\title{
SPINORIAL CLASSIFICATION OF SPIN(7) STRUCTURES
}

\author{
LUCÍA MARTÍN-MERCHÁN
}

Abstract. We describe the different classes of $\operatorname{Spin}(7)$ structures in terms of spinorial equations. We relate them to the spinorial description of $\mathrm{G}_{2}$ structures in some geometrical situations. Our approach enables us to analyze invariant $\operatorname{Spin}(7)$ structures on quasi abelian Lie algebras.

\section{INTRODUCTION}

Berger's list [2] (1955) of possible holonomy groups of simply connected, irreducible and non-symmetric Riemannian manifolds contains the so-called exceptional holonomy groups, $\mathrm{G}_{2}$ and Spin(7), which occur in dimensions 7 and 8 respectively. Non-complete metrics with exceptional holonomy were given by Bryant in [3], complete metrics were obtained by Bryant and Salamon in [4], but compact examples were not constructed until 1996, when Joyce published [12], [13] and [14].

The remaining groups of Berger's list different from SO(n), called special holonomy groups, are U(n), $\mathrm{SU}(\mathrm{n}), \operatorname{Sp}(\mathrm{n})$ and $\mathrm{Sp}(\mathrm{n}) \cdot \mathrm{Sp}(1)$. If the holonomy of a Riemannian manifold is contained in a group $\mathrm{G}$, the manifold admits a $\mathrm{G}$ structure, that is, a reduction to $\mathrm{G}$ of its frame bundle. Therefore, holonomy is homotopically obstructed by the presence of $\mathrm{G}$ structures. Examples of manifolds endowed with $\mathrm{G}$ structures for some of the holonomy groups in the Berger list are not only easier to obtain than manifolds with holonomy in G, but also relevant in M-theory, especially if they admit a characteristic connection [10], that is, a metric connection with totally skew-symmetric torsion whose holonomy is contained in G. It is worth mentioning that Ivanov proved in [11] that each manifold with a Spin(7) structure admits a unique characteristic connection. Moreover, Friedrich proved in [9] that Spin(7) is the unique compact simple Lie group $\mathrm{G}$ such that all the $\mathrm{G}$ structures admit a unique characteristic connection.

The Lie group $\mathrm{G}_{2}$ is compact, simple and simply connected. It consists of the endomorpisms of $\mathbb{R}^{7}$ which preserve the cross product from the imaginary part of the octonions [22]. Hence, a $\mathrm{G}_{2}$ structure on a manifold $Q$ determines a 3 -form $\Psi$, a metric and an orientation. In [7], Fernández and Gray classify $\mathrm{G}_{2}$ structures into 16 different classes in terms of the $\mathrm{G}_{2}$ irreducible components of $\nabla \Psi$. Related to this, the analysis of the intrinsic torsion in [5] allowed to obtain equations involving $d \Psi$ and $d(* \Psi)$ for each of the 16 classes, determined by the $\mathrm{G}_{2}$ irreducible components of $\Lambda^{4} T^{*} Q$ and $\Lambda^{5} T^{*} Q$. In particular, one obtains that the holonomy of $Q$ is contained in $\mathrm{G}_{2}$ if and only if $d \Psi=0$ and $d(* \Psi)=0$. The Lie group $\operatorname{Spin}(7)$ is also compact, simple and simply connected. It is the group of endomorphisms of $\mathbb{R}^{8}$ which preserve the triple cross product from the octonions [22]. Thus, a Spin(7) structure on a manifold $M$ determines 4 -form $\Omega$, a metric and an orientation. In [6], Fernández classifies $\operatorname{Spin}(7)$ structures into 4 classes in terms of differential equations for $d \Omega$, which are determined by the Spin $(7)$-irreducible components of $\Lambda^{5} T^{*} M$. Parallel structures verify $d \Omega=0$, locally conformally parallel structures satisfy $d \Omega=\theta \wedge \Omega$ for a closed 1 -form $\theta$ and balanced structures verify $*(d \Omega) \wedge \Omega=0$. A generic $\operatorname{Spin}(7)$ structure, which does not satisfy any of the previous conditions, is called mixed.

The relationship between $\mathrm{G}_{2}$ and $\operatorname{Spin}(7)$ structures was firstly explored by Martín-Cabrera in [17]. Each oriented hypersurface of a manifold equipped with a $\operatorname{Spin}(7)$ structure naturally inherits a $\mathrm{G}_{2}$ structure whose type is determined by the $\operatorname{Spin}(7)$ structure of the ambient manifold and some extrinsic information of the submanifold, such as the Weingarten operator. Following the same viewpoint, Martín-Cabrera constructed $\operatorname{Spin}(7)$ structures on $S^{1}$-principal bundles over $\mathrm{G}_{2}$ manifolds in [18]. Both approaches allowed to construct manifolds with $\mathrm{G}_{2}$ and $\operatorname{Spin}(7)$ structures of different pure types.

It turns out that manifolds admitting $\mathrm{SU}(3), \mathrm{G}_{2}$ and $\mathrm{Spin}(7)$ structures are spin and their spinorial bundle has a unitary section $\eta$ which determines the structure. In [1], spinorial formalism was used to deal with the distinct aspects of $\mathrm{SU}(3)$ and $\mathrm{G}_{2}$ structures, such as the classification of both types of structures, $\mathrm{SU}(3)$ structures on hypersurfaces of $\mathrm{G}_{2}$ manifolds and different types of Killing spinors. A

2010 Mathematics Subject Classification. Primary 53C27. Secondary: 53C10, 22E25.

Key words and phrases. Spin(7)-structures, Spinor, Intrinsic torsion, Characteristic connection, $\mathrm{G}_{2}$ distributions, Quasi abelian Lie algebras. 
clear advantage of this viewpoint is that a unique object, the spinor, encodes the whole geometry of the structure. For instance, a $\mathrm{G}_{2}$ structure on a Riemannian manifold $(Q, g)$ with associated 3 -form $\Psi$ is determined by a suitable spinor $\eta$ according to the formula $\Psi(X, Y, Z)=(X \eta, Y Z \eta)$ where $(\cdot, \cdot)$ denotes the scalar product in the spinorial bundle and juxtaposition of vectors indicates the Clifford product. Any oriented hypersurface $Q^{\prime}$ with normal vector field $N$ inherits an SU(3) structure implicitly defined by $\Psi=N^{*} \wedge \omega+\operatorname{Re}(\Theta)$, where $N^{*}(X)=g(N, X)$ for $X \in T Q$. But both the Kahler form $\omega$ and the $(3,0)$-form $\operatorname{Re}(\Theta)$ turn out to be determined by the same spinor $\eta$.

In this paper we follow the ideas of [1] to describe the geometry of Spin(7) structures from a spinorial viewpoint, starting from the classification of these structures, continuing to analyze the relationship between $\mathrm{G}_{2}$ and $\operatorname{Spin}(7)$ structures and finishing with the study of invariant $\operatorname{Spin}(7)$ structures on quasi abelian Lie algebras.

Our first result, Theorem 4.5 in section 4, describes each type of $\operatorname{Spin}(7)$ structure with spinorial equations. To state it, we have to mention that if the structure is determined by a spinor $\eta$ and $R$ is a $\operatorname{Spin}(7)$ reduction of the frame bundle of the manifold, there is a natural isomorphism $c: R \times \operatorname{Spin}(7)$ $\mathfrak{s p i n}(7)^{\perp} \rightarrow\langle\eta\rangle^{\perp}$ (see Lemma 3.1, section 3 for details).

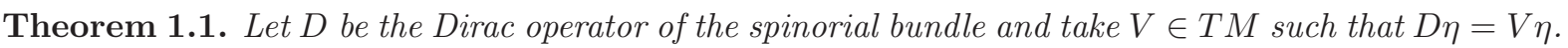
The $\operatorname{Spin}(7)$ structure $\Omega$ defined by $\eta$ is:

1. Parallel if $\nabla \eta=0$.

2. Locally conformally parallel if $i(V) \Omega=28$ alt $\left(c^{-1} \nabla \eta\right)$.

3. Balanced if $D \eta=0$.

Our techniques also allow us to identify the intrinsic torsion of the structure and to obtain the formula for the unique characteristic connection of each $\operatorname{Spin}(7)$ structure, given by Ivanov in [11, Theorem 1.1].

We also introduce the concept of $\mathrm{G}_{2}$ distributions, a general setting to relate $\mathrm{G}_{2}$ and $\operatorname{Spin}(7)$ structures.

Definition 1.2. Let $(M, g)$ be an oriented 8-dimensional Riemannian manifold and let $\mathcal{D}$ be a cooriented distribution of codimension 1 . We say that $\mathcal{D}$ has a $\mathrm{G}_{2}$ structure if the principal $\mathrm{SO}(7)$ bundle $P(\mathcal{D})$ is spin and the spinorial bundle $\Sigma(\mathcal{D})$ admits a unitary section.

This construction allows us to obtain the results which appear in [17] and [18] about $\mathrm{G}_{2}$ structures on hypersurfaces of $\operatorname{Spin}(7)$ manifolds and $S^{1}$-principal bundles over $\mathrm{G}_{2}$ manifolds. Related to this, we also study warped products of manifolds admitting a $G_{2}$ structure with $\mathbb{R}$.

The formalism of $\mathrm{G}_{2}$ distributions enables us to study invariant $\mathrm{Spin}(7)$ structures on quasi-abelian Lie algebras, that is, Lie algebras with a codimension 1 abelian ideal. To state the result, which is Theorem 7.7, suppose that the Lie algebra is $\mathfrak{g}=\left\langle e_{0}, \ldots, e_{7}\right\rangle$ with abelian ideal $\mathbb{R}^{7}=\left\langle e_{1}, \ldots, e_{7}\right\rangle$ and it is endowed with the canonical metric and volume form.

Theorem 1.3. Denote by $\mathcal{E}=\left.a d\left(e_{0}\right)\right|_{\mathbb{R}^{7}}$ and let $\mathcal{E}_{13}$ and $\mathcal{E}_{24}$ be the symmetric and skew-symmetric parts of the endomorphism. Then, $\mathfrak{g}$ admits a $\operatorname{Spin}(7)$ structure of type:

1. Parallel, if and only if $\mathcal{E}_{13}=0$ and the eigenvalues of $\mathcal{E}_{24}$ are $0, \pm \lambda_{1} i, \pm \lambda_{2} i, \pm\left(\lambda_{1}+\lambda_{2}\right) i$, for some $0 \leq \lambda_{1} \leq \lambda_{2}$.

2. Locally conformally parallel and non-parallel if and only if $\mathcal{E}_{13}=h \mathrm{Id}$ with $h \neq 0$ and the eigenvalues of $\mathcal{E}_{24}$ are $0, \pm \lambda_{1} i, \pm \lambda_{2} i, \pm\left(\lambda_{1}+\lambda_{2}\right) i$, for some $0 \leq \lambda_{1} \leq \lambda_{2}$.

3. Balanced if and only if $\mathfrak{g}$ is unimodular and the eigenvalues of $\mathcal{E}_{24}$ are $0, \pm \lambda_{1} i, \pm \lambda_{2} i, \pm\left(\lambda_{1}+\lambda_{2}\right) i$, for some $0 \leq \lambda_{1} \leq \lambda_{2}$.

Moreover, if $\mathcal{E}_{24} \neq 0$ then it admits a $\operatorname{Spin}(7)$ structure of mixed type.

From this, it follows (Corollary 7.8) that there are no quasi abelian solvmanifolds which admit a locally conformally parallel Spin(7) structure. In addition, this result allows us to give an example of a nilmanifold admitting both an invariant balanced structure and an invariant mixed structure. A compact manifold admitting a parallel structure is also obtained as a quotient of a simply connected Lie group whose Lie algebra is quasi abelian. Despite not being diffeomorphic to a torus, it is flat. Indeed, we prove that quasi abelian Lie algebras which admit an invariant Spin(7) parallel structure are flat (Corollary 7.9).

This paper is organized as follows. Section 2 contains a review of algebraic aspects of Spin(7) geometry. Section 3 identifies the instrinsic torsion of the Levi-Civita connection with a spinor, section 4 is devoted to obtain the classification of $\operatorname{Spin}(7)$ structures in terms of spinors and section 5 provides an alternative proof of the existence of the characteristic connection. Section 6 provides a complete analysis of $\mathrm{G}_{2}$ structures on distributions and then focuses on the particular cases described above. Finally, section 7 deals with invariant structures on quasi abelian Lie algebras and provides compact examples. 


\section{Preliminaries}

In this section we introduce some aspects of Clifford algebras, 8-dimensional spin manifolds and Spin(7) representations, which can be found in [8], [15] and [22] as well as the notations that we will use in the sequel.

2.1. Spin(7) structures. Let $(M, g)$ be an oriented Riemannian 8-manifold and let $P(M)$ be the associated $\mathrm{SO}(8)$ frame bundle. Provided that $M$ is spin, that is $w_{2}(M)=0$, we can take a $\operatorname{Spin}(8)$ principal bundle $\tilde{P}(M)$ over $M$ which is a double covering $\pi: \tilde{P}(M) \rightarrow P(M)$ equivariant under the adjoint action $A d: \operatorname{Spin}(8) \rightarrow \mathrm{SO}(8)$. We may also denote by $A d$ the induced action of $\operatorname{Spin}(8)$ on $T M$.

The associated spinorial bundle is $\Sigma(M)=\tilde{P}(M) \times_{\rho} \mathbb{R}^{16}$ where we have denoted by $\rho: \operatorname{Spin}(8) \rightarrow$ $\mathrm{SO}(16)$ the real spinorial representation, constructed by restricting the isomorphism $\mathrm{Cl}_{8} \cong \mathrm{GL}(16)$ and equipping $\mathbb{R}^{16}$ with a metric $(\cdot, \cdot)$ which makes the Clifford product a skew-symmetric endomorphism. The induced metric on $\Sigma(M)$ will be denoted in the same way, and the elements of this bundle by $\phi=[\tilde{F}, v]$, where $\tilde{F} \in \tilde{P}(M)$ and $v \in \mathbb{R}^{16}$.

The Clifford multiplication with a vector field is extended to an action of $\Lambda^{k} T^{*} M$ defined as follows.

1. The product with a covector is defined by $X^{*} \phi=X \phi$, where we used the canonical identification between the tangent and the cotangent bundle: $X^{*}=g(X, \cdot)$.

2. If the product is defined on $\Lambda^{\ell} T^{*} M$ when $\ell \leq k$, we define

$$
\left(X^{*} \wedge \beta\right) \phi=X(\beta \phi)+(i(X) \beta) \phi,
$$

where $i(X) \beta$ denotes the contraction, $\beta \in \Lambda^{k} T^{*} M$ and $X \in T M$. This product is extended lineary to $\Lambda^{k+1} T^{*} M$.

For instance, we have:

$$
\begin{aligned}
& \left(X^{*} \wedge Y^{*}\right) \phi=(X Y+g(X, Y)) \phi, \\
& \left(X^{*} \wedge Y^{*} \wedge Z^{*}\right) \phi=(X Y Z+g(X, Y) Z-g(X, Z) Y+g(Y, Z) X) \phi .
\end{aligned}
$$

The volume form $\nu_{8}$ of $\mathbb{R}^{8}$ provides $\mathbb{R}^{16}$ with a $\operatorname{Spin}(8)$ equivariant endomorphism:

$$
\nu_{8}: \mathbb{R}^{16} \rightarrow \mathbb{R}^{16}, \quad \phi \longmapsto \nu_{8} \phi .
$$

Since $\nu_{8}^{2}=1$, there is a splitting $\mathbb{R}^{16}=\Delta^{+} \oplus \Delta^{-}$where $\Delta^{ \pm}$is the eigenspace associated to \pm 1 . Therefore, $\Sigma(M)=\Sigma(M)^{+} \oplus \Sigma(M)^{-}$, where $\Sigma(M)^{ \pm}=\tilde{P}(M) \times_{\rho} \Delta^{ \pm}$. Also note that $X\left(\Sigma(M)^{ \pm}\right) \subset \Sigma(M)^{\mp}$ if $X \in \mathfrak{X}(M)$.

At each $p \in M$, the action $\operatorname{Spin}(8) \rightarrow \operatorname{SO}\left(\Sigma_{p}(M)^{+}\right), \tilde{\varphi}[\tilde{F}, v]=[\tilde{F}, \rho(\tilde{\varphi}) v]$ is a double covering, so that the existence of a unitary spinor $\eta \in \Gamma\left(\Sigma(M)^{+}\right)$determines an identification between $\operatorname{Spin}(7)$ and the stabilizer of $\eta_{p}, \operatorname{Stab}\left(\eta_{p}\right)$. Besides, the restriction $A d: \operatorname{Spin}(8) \rightarrow \operatorname{SO}\left(T_{p} M\right)$ to $\operatorname{Stab}\left(\eta_{p}\right)$ is injective since $\operatorname{ker}(A d)=\{1,-1\}$ and $-1 \notin \operatorname{Stab}\left(\eta_{p}\right)$.

The previous considerations allow us to define a 4 -form $\Omega$ on $M$ such that $\operatorname{Ad}\left(\operatorname{Stab}\left(\eta_{p}\right)\right)=\operatorname{Stab}\left(\Omega_{p}\right)$. Indeed, observe that there is a well defined map:

$$
T M \times T M \times T M \rightarrow T M, \quad(X, Y, Z) \longmapsto X \times Y \times Z \text { s.t },(X \times Y \times Z) \eta=\left(X^{*} \wedge Y^{*} \wedge Z^{*}\right) \eta,
$$

which turns out to be a positive triple product, that is, it verifies [22, Definition 6.1]:

1. The vector $X \times Y \times Z$ is perpendicular to $X, Y$ and $Z$.

2. $\|X \times Y \times Z\|=\left\|X^{*} \wedge Y^{*} \wedge Z^{*}\right\|$.

3. If we take orthonormal vectors $W, X, Y, Z$ such that $W$ is perpendicular to $X \times Y \times Z$, then $X \times Y \times(X \times Z \times W)=Y \times Z \times W$.

The first property follows from (2) and the second one is obvious. To check the third one we observe that $Y$ is perpendicular to $X \times Z \times W$ since $g(W, X \times Y \times Z)=(W \eta, X Y Z \eta)=(Y \eta, X Z W \eta)=$ $g(Y, X \times Z \times W)$, and therefore:

$$
X \times Y \times(X \times Z \times W) \eta=X Y X Z W \eta=Y Z W \eta=(Y \times Z \times W) \eta .
$$

Definition 2.1. The associated 4 -form to the triple cross product is:

$$
\begin{aligned}
\Omega(W, X, Y, Z) & =((X \times Y \times Z) \eta, W \eta)=((X Y Z+g(X, Y) Z-g(X, Z) Y+g(X, Y) Z) \eta, W \eta) \\
& =\frac{1}{2}((-W X Y Z+W Z Y X) \eta, \eta) .
\end{aligned}
$$

Since $\tilde{\varphi}(X \phi)=\operatorname{Ad}(\tilde{\varphi})(X)(\tilde{\varphi} \phi)$ if $\tilde{\varphi} \in \operatorname{Spin}(8), X \in T M$ and $\phi \in \Sigma(M)$, it is not hard to check that $\operatorname{Stab}\left(\eta_{p}\right)=\operatorname{Stab}\left(\Omega_{p}\right)$. Some important properties of this form are the following: 
1. If $\left(X_{0}, \ldots, X_{7}\right)$ is an orthonormal oriented basis and $\sigma$ is a permutation then $* \Omega=\Omega$ since $X_{\sigma(0)} X_{\sigma(1)} X_{\sigma(2)} X_{\sigma(3)} \eta=(-1)^{\operatorname{sgn}(\sigma)} X_{\sigma(4)} X_{\sigma(5)} X_{\sigma(6)} X_{\sigma(7)} \eta$.

2. Given orthonormal vector fields $e_{0}, e_{1}, e_{2}, e_{4}$ such that $e_{4}$ is perpendicular to $e_{3}=e_{0} \times e_{1} \times e_{2}$, we can find [22, Theorem 7.12] an orthonormal frame $\left(e_{0}, \ldots, e_{7}\right)$ such that:

$$
\begin{aligned}
\Omega= & e^{0123}-e^{0145}-e^{0167}-e^{0246}+e^{0257}-e^{0347}-e^{0356} \\
& +e^{4567}-e^{2367}-e^{2345}-e^{1357}+e^{1346}-e^{1256}-e^{1247},
\end{aligned}
$$

where we have used the short-hand notation $e^{i}$ for $g\left(e_{i}, \cdot\right)$ and $e^{i j k l}$ for $e^{i} \wedge e^{j} \wedge e^{k} \wedge e^{l}$. We will also denote the Clifford product $e_{i} e_{j}$ by $e_{i j}$ and so on. A frame of this type will be called a Cayley frame. Since those frames verify $\left(e_{0} \cdots e_{7}\right) \eta=\eta$, they are positively oriented.

2.2. $\operatorname{Spin}(7)$ representations. Let us denote the standard basis of $\mathbb{R}^{8}$ by $\left(e_{0}, \ldots, e_{7}\right)$, and the standard $\operatorname{Spin}(7)$ structure of $\mathbb{R}^{8}$ by $\Omega_{0}$, given by (3).

The canonical representation of $\operatorname{Spin}(7)=\operatorname{Stab}\left(\Omega_{0}\right) \subset \operatorname{SO}(8)$ on $\Lambda^{k} \mathbb{R}^{8}$ induces an orthogonal decomposition of this space into irreducible $\operatorname{Spin}(7)$ invariant subspaces. The expression $\Lambda_{l}^{k} \mathbb{R}^{8}$ denotes such an $l$-dimensional subspace of $\Lambda^{k} \mathbb{R}^{8}$. Observe that Hodge star operator $*$ gives isomorphisms between $\Lambda^{k} \mathbb{R}^{8}$ and $\Lambda^{8-k} \mathbb{R}^{8}$ determining that $\Lambda_{l}^{k} \mathbb{R}^{8}=* \Lambda_{l}^{8-k} \mathbb{R}^{8}$ if $k \leq 4$. We are going to describe briefly the splitting at degrees $k=2$ and $k=3$ but a complete proof can be found in [22, Theorem 9.8]. The decomposition goes as follows:

$$
\begin{aligned}
\Lambda^{2} \mathbb{R}^{8} & =\Lambda_{7}^{2} \mathbb{R}^{8} \oplus \Lambda_{21}^{2} \mathbb{R}^{8}, \\
\Lambda^{3} \mathbb{R}^{8} & =\Lambda_{8}^{3} \mathbb{R}^{8} \oplus \Lambda_{48}^{3} \mathbb{R}^{8} .
\end{aligned}
$$

The first one comes from the orthogonal splitting $\Lambda^{2} \mathbb{R}^{8}=\mathfrak{s o}(8)=\mathfrak{s p i n}(7) \oplus \mathfrak{m}$, where $\mathfrak{m}=\mathfrak{s p i n}(7)^{\perp}$. An alternative description may be done by considering the map:

$$
\Lambda^{2} \mathbb{R}^{8} \rightarrow \Lambda^{2} \mathbb{R}^{8}, \quad \beta \longmapsto *\left(\beta \wedge \Omega_{0}\right),
$$

which is $\operatorname{Spin}(7)$-equivariant, symmetric and traceless. Therefore, $\Lambda^{2} T^{*} M$ splits into eigenspaces which must coincide with the previous ones due to the irreducibility. It can be checked that the eigenvalues are 3 on $\Lambda_{7}^{2} \mathbb{R}^{8}$ and -1 on $\Lambda_{21}^{2} \mathbb{R}^{8}$. Moreover, the set $\left\{\alpha_{j}=e^{0 j}-i\left(e_{0}\right) i\left(e_{j}\right) \Omega_{0}\right\}_{j=1}^{7}$ is a basis of $\Lambda_{7}^{2} T^{*} M$.

The subspaces involved in the second splitting are:

$$
\begin{aligned}
\Lambda_{8}^{3} \mathbb{R}^{8} & =i\left(\mathbb{R}^{8}\right) \Omega_{0}, \\
\Lambda_{48}^{3} \mathbb{R}^{8} & =\operatorname{ker}\left(\cdot \wedge \Omega_{0}: \Lambda^{3} \mathbb{R}^{8} \rightarrow \Lambda^{7} \mathbb{R}^{8}\right) .
\end{aligned}
$$

Finally, a $\operatorname{Spin}(7)$ structure on the Riemannian manifold $(M, g)$ determines a canonical splitting of $\Lambda^{k} T^{*} M$. If we take the $\operatorname{Spin}(7)$ reduction $R$ of the $\mathrm{SO}(8)$ principal bundle given by the Cayley frames, then those are given by $\Lambda_{l}^{k} T^{*} M=R \times_{\operatorname{Spin}(7)} \Lambda_{l}^{k} \mathbb{R}^{8}$.

\section{THE INTRINSIC TORSION}

We are going to compute the intrinsic torsion of the Levi-Civita connection, $\Gamma \in T M \otimes \Lambda_{7}^{2} T^{*} M$. Recall that the Levi-Civita connection $\nabla$ on $T M$ induces a connection $\omega$ on $P(M)$. Then a connection on the $\operatorname{Spin}(7)$ reduction $R$ is defined by $\omega^{\prime}=\left.p(\omega)\right|_{T R}$, where $p$ denotes the orthogonal projection to $\mathfrak{s p i n}(7)$. The connection that $\omega^{\prime}$ induces on $T M$ is denoted by $\nabla^{\prime}$ and determines the intrinsic torsion by means of the expression:

$$
\nabla_{X} Y=\nabla_{X}^{\prime} Y+\Gamma(X) Y .
$$

The skew-symmetric endomorphism $\Gamma(X)$ can be identified with a 2-form which lies in $R \times{ }_{\operatorname{Spin}(7)} \mathfrak{m}=$ $\Lambda_{7}^{2} T^{*} M$ for each $X \in T M$. To compute it, we will first prove that the vector bundles $\Lambda_{7}^{2} T^{*} M$ and $H=\langle\eta\rangle^{\perp}$ are isomorphic:

Lemma 3.1. There is a well defined $\operatorname{Spin}(7)$-equivariant map

$$
\Lambda^{2} T^{*} M \rightarrow H, \quad \alpha \longmapsto \alpha \eta,
$$

whose kernel is $\Lambda_{21}^{2} T^{*} M$. Indeed, its restriction $c: \Lambda_{7}^{2} T^{*} M \rightarrow H$ is an isomorphism whose inverse is given by $\left(c^{-1} \phi\right)(X, Y)=\frac{1}{4}(\phi,(X Y+g(X, Y)) \eta)$. 
Proof. The spinor $\beta \eta$ is perpendicular to $\eta$ if $\beta \in \Lambda^{2} T^{*} M$. Therefore, the map is well-defined and it is $\operatorname{Spin}(7)$-equivariant since $\operatorname{Spin}(7)=\operatorname{Stab}\left(\eta_{p}\right)$.

To prove that $c$ is an isomorphism, we first claim that if $\left(e_{0}, \ldots, e_{7}\right)$ is a Cayley frame then $\alpha_{j} \eta=$ $4 e^{0 j} \eta$. Observe that we only need to check this formula for $j=1$ since $c$ is $\operatorname{Spin}(7)$-equivariant and $\mathrm{G}_{2}=\operatorname{Spin}(7) \cap \operatorname{Stab}\left(e_{0}\right)$ acts transitively on the 6 -sphere generated by $\left(e_{1}, \ldots, e_{7}\right)$. In this case, $\alpha_{1}=$ $e^{01}+e^{23}-e^{45}-e^{67}$ and if $(i, j) \in\{(2,3),(5,4),(7,6)\}$ we have that $\Omega\left(e_{0}, e_{1}, e_{i}, e_{j}\right)=1$. The previous equality means that $e_{0} \eta=e_{1 i j} \eta$, so that $e^{01} \eta=e^{i j} \eta$.

Moreover, since $\left\{e^{0 i} \eta\right\}_{i=1}^{7}$ is an orthonormal basis of $H$ we have that

$$
c^{-1}(\phi)=\frac{1}{4} \sum_{i=1}^{7}\left(\phi, e^{0 i} \eta\right) \alpha_{i} .
$$

If $X=e_{0}, Y=e_{1}$ are orthonormal vectors then $\alpha_{j}\left(e_{0}, e_{1}\right)=\left(e^{0 j}-i\left(e_{0}\right) i\left(e_{j}\right) \Omega\right)\left(e_{0}, e_{1}\right)=\delta_{j 1}$. Hence, $c^{-1} \phi\left(e_{0}, e_{1}\right)=\frac{1}{4}\left(\phi, e_{0} e_{1} \eta\right)$.

Finally, by dimensional reasons the Clifford product with $\eta$ must vanish on $\Lambda_{21}^{2} T^{*} M$.

The previous result enables us to find a formula for the intrinsic torsion:

Proposition 3.2. The intrinsic torsion is given by $\Gamma(X)=2 c^{-1} \nabla_{X} \eta$.

Proof. We also denote by $\nabla$ and $\nabla^{\prime}$ the induced connections on the spinorial bundle. According to [8, p. 60] we have that:

$$
\nabla_{X} \phi=\nabla_{X}^{\prime} \phi+\frac{1}{2} \Gamma(X) \phi
$$

where $\Gamma(X)$ acts on $\phi$ as a 2 -form. Since the holonomy of the connection $\nabla^{\prime}$ is contained in $\operatorname{Spin}(7)$ and $\operatorname{Stab}\left(\eta_{p}\right)=\operatorname{Spin}(7)$ we have that $\nabla^{\prime} \eta=0$. Finally, if $X \in T M$ then $\nabla_{X} \eta \in H$ and $\Gamma(X) \in R \times_{\operatorname{Spin}(7)} \mathfrak{m}$ thus, Lemma 3.1 shows that $\Gamma(X)=2 c^{-1} \nabla_{X} \eta$.

\section{Spinorial Classification of $\operatorname{Spin}(7)$ Structures}

Spin structures are classified [6] according to the $\operatorname{Spin}(7)$ irreducible parts of $* d \Omega$ on $\Lambda^{3} T^{*} M$ in the following pure types:

Definition 4.1. A Spin(7)-structure given by $\Omega$ is said to be:

1. Parallel, if $* d \Omega=0$.

2. Locally conformally parallel, if $* d \Omega \in \Lambda_{8}^{3} T^{*} M$.

3. Balanced if $* d \Omega \in \Lambda_{48}^{3} T^{*} M$.

The Lee form of $\Omega$ is the unique $\theta \in \Lambda^{1} T^{*} M$ such that the orthogonal projection to $\Lambda_{8}^{5} T^{*} M$ of $d \Omega$ is $\theta \wedge \Omega$.

Remark 4.2. Suppose that the structure is locally conformally parallel. Let $O$ be a contractible open set, take a primitive $f$ of $-\left.\frac{1}{4} \theta\right|_{O}$ and define the metric $g^{\prime}=\left.e^{2 f} g\right|_{O}$. The associated $\operatorname{Spin}(7)$ structure is $\Omega^{\prime}=\left.e^{4 f} \Omega\right|_{O}$ and it verifies $d \Omega^{\prime}=0$. Therefore, $\left.\Omega\right|_{O}$ is conformal to a parallel structure. This justifies the name.

In order to rewrite this classification by means of $\eta$, we are going to calculate $* d \Omega$. For this purpose, consider the Dirac operator $D$ at $\Sigma(M)$ and the vector field $V$ such that

$$
D \eta=V \eta \text {. }
$$

Then, the 3 -form $\gamma_{8}(X, Y, Z)=(D \eta,(X \times Y \times Z) \eta)=(i(V) \Omega)(X, Y, Z)$ obviously lies in $\Lambda_{8}^{3} T^{*} M$.

Proposition 4.3. Using the previous notation, we have:

$$
* d \Omega=2\left(\gamma_{8}-12 \operatorname{alt}\left(c^{-1} \nabla \eta\right)\right),
$$

where $\operatorname{alt}(\mathrm{T})\left(X_{1}, \ldots, X_{n}\right)=\frac{1}{n !} \sum_{\sigma \in S_{n}}(-1)^{\operatorname{sgn} \sigma} \mathrm{T}\left(X_{\sigma(1)}, \ldots, X_{\sigma(n)}\right)$ if $\mathrm{T}$ is a section of $\otimes^{n} T M$.

Proof. Since $\nabla$ is a metric connection on the spinorial bundle and acts as a derivation for the Clifford product, we get:

$$
\begin{aligned}
\left(\nabla_{T} \Omega\right)(W, X, Y, Z) & =\frac{1}{2}\left(\left((-W X Y Z+W Z Y X) \nabla_{T} \eta, \eta\right)+\left((-W X Y Z+W Z Y X) \eta, \nabla_{T} \eta\right)\right) \\
& =\frac{1}{2}\left((-Z Y X W+X Y Z W-W X Y Z+W Z Y X) \eta, \nabla_{T} \eta\right) .
\end{aligned}
$$


Take orthonormal vectors $X, Y, Z$ and an orthonormal oriented basis $\left(X_{0}, \ldots X_{7}\right)$ such that $X_{0}=X$, $X_{1}=Y$ and $X_{2}=Z$. Then,

$$
\begin{aligned}
\delta \Omega(X, Y, Z) & =-\sum_{i=3}^{7} \nabla_{X_{i}} \Omega\left(X_{i}, X, Y, Z\right)=-2 \sum_{i=3}^{7}\left(X Y Z \eta, X_{i} \nabla_{X_{i}} \eta\right) \\
& =-2(D \eta,(X \times Y \times Z) \eta)+2\left(X Y Z \eta, X \nabla_{X} \eta+Y \nabla_{Y} \eta+Z \nabla_{Z} \eta\right) \\
& =-2\left((D \eta,(X \times Y \times Z) \eta)-\left(Y Z \eta, \nabla_{X} \eta\right)+\left(X Z \eta, \nabla_{Y} \eta\right)-\left(X Y \eta, \nabla_{Z} \eta\right)\right. \\
& =-2\left((D \eta,(X \times Y \times Z) \eta)-12 \operatorname{alt}\left(c^{-1} \nabla \eta\right)(X, Y, Z)\right) .
\end{aligned}
$$

Note that the coefficient 12 comes from the normalization of alt and the expression $c^{-1}\left(\nabla_{X} \eta\right)(X, Y)=$ $\frac{1}{4}\left((X Y+g(X, Y)) \eta, \nabla_{X} \eta\right)$.

We are going to decompose $* d \Omega$ according to the previous splitting.

Lemma 4.4. The 3 -form $\gamma_{48}=3 \gamma_{8}-84 \operatorname{alt}\left(c^{-1} \nabla \eta\right)$ lies in $\Lambda_{48}^{3} T^{*} M$ and

$$
* d \Omega=\frac{2}{7} \gamma_{48}+\frac{8}{7} \gamma_{8}
$$

Proof. Take a unitary vector $X$ and a Cayley frame $\left(e_{0}, e_{1}, \ldots, e_{7}\right)$ such that $X=e_{0}$. Then:

$$
\begin{aligned}
\left(\gamma_{8} \wedge \Omega\right)\left(e_{1}, \ldots, e_{7}\right)= & \left(D \eta,\left(e_{123}-e_{145}-e_{167}-e_{246}+e_{257}-e_{347}-e_{356}\right) \eta\right) \\
= & 7\left(D \eta, e_{0} \eta\right)=7 V^{*}(X), \\
\left(12 \operatorname{alt}\left(c^{-1} \nabla \eta\right) \wedge \Omega\right)\left(e_{1}, \ldots, e_{7}\right)= & \mathfrak{S}\left(\nabla_{e_{1}} \eta, e_{23} \eta\right)-\mathfrak{S}\left(\nabla_{e_{1}} \eta, e_{45} \eta\right)-\mathfrak{S}\left(\nabla_{e_{1}} \eta, e_{67} \eta\right) \\
& -\mathfrak{S}\left(\nabla_{e_{2}} \eta, e_{46} \eta\right)+\mathfrak{S}\left(\nabla_{e_{2}} \eta, e_{57} \eta\right)-\mathfrak{S}\left(\nabla_{e_{3}} \eta, e_{47} \eta\right) \\
& -\mathfrak{S}\left(\nabla_{e_{3}} \eta, e_{56} \eta\right)=3\left(D \eta, e_{0} \eta\right)=3 V^{*}(X) .
\end{aligned}
$$

We denoted by $\mathfrak{S}$ the cyclic sums in the indices involved. To arrange the last term observe that each index appears 3 times and:

$$
\begin{aligned}
\mathfrak{S}\left(\nabla_{e_{1}} \eta, e_{23} \eta\right) & =\left(e_{1} \nabla_{e_{1}} \eta+e_{2} \nabla_{e_{2}} \eta+e_{3} \nabla_{e_{3}} \eta, e_{123} \eta\right)=\left(e_{1} \nabla_{e_{1}} \eta+e_{2} \nabla_{e_{2}} \eta+e_{3} \nabla_{e_{3}} \eta, e_{0} \eta\right), \\
-\mathfrak{S}\left(\nabla_{e_{1}} \eta, e_{45} \eta\right) & =\left(e_{1} \nabla_{e_{1}} \eta+e_{4} \nabla_{e_{4}} \eta+e_{5} \nabla_{e_{5}} \eta,-e_{145} \eta\right)=\left(e_{1} \nabla_{e_{1}} \eta+e_{4} \nabla_{e_{4}} \eta+e_{5} \nabla_{e_{5}} \eta, e_{0} \eta\right),
\end{aligned}
$$

and so on. Note that we have used, as in the proof of Lemma 3.1, that $e_{123} \eta=e_{0} \eta=-e_{145} \eta$.

Since Cayley bases are positive oriented, we get $*\left(V^{*}\right)=\frac{1}{7}\left(\gamma_{8} \wedge \Omega\right)=4 \operatorname{alt}\left(c^{-1} \nabla \eta\right)$, so that $\gamma_{48}$ as defined above lies in $\Lambda_{48}^{3} T^{*} M$. Finally, taking into account the formula for $* d \Omega$ in Proposition 4.3, we get $* d \Omega=\frac{2}{7} \gamma_{48}+\frac{8}{7} \gamma_{8}$.

We can now rewrite the classification of $\operatorname{Spin}(7)$ structures:

Theorem 4.5. The $\operatorname{Spin}(7)$ structure given by $\Omega$ is:

1. Parallel if $\nabla \eta=0$.

2. Locally conformally parallel if $i(V) \Omega=28$ alt $\left(c^{-1} \nabla \eta\right)$.

3. Balanced if $D \eta=0$.

Moreover, the Lee form is given by $\theta=\frac{8}{7} V^{*}$, where $V$ is defined as in the equation (4).

Proof. It is an immediate consequence of Definition 4.1 and Lemma 4.4. To compute the Lee form we have used that the projection of $d \Omega$ to $\Lambda_{8}^{5} T^{*} M$ is $-\frac{8}{7} * \gamma_{8}$ and the formula $i(X) \Omega=*\left(X^{*} \wedge \Omega\right)$, which can be easily checked taking a Cayley frame and $X=e_{0}$.

\section{THE CHARACTERISTIC CONNECTION.}

The characteristic connection of a $\operatorname{Spin}(7)$ structure is a connection $\nabla^{c}$ with totally skew-symmetric torsion, such that $\nabla^{c} \Omega=0$. The computations above allow us to prove the existence and uniqueness of the characteristic connection for manifolds with a $\operatorname{Spin}(7)$ structure. This is a well known result which appears in [11, Theorem 1.1]. Our proof is based on the argument of Theorem 3.1 in [9].

Consider the Spin(7)-equivariant maps which are given in terms of a local Cayley frame:

$$
\begin{aligned}
& \Theta: \Lambda^{3} T^{*} M \rightarrow T M \otimes \Lambda_{7}^{2} T^{*} M, \quad \beta \longmapsto \Theta(\beta)=\sum_{j=0}^{7} e_{j} \otimes p_{7}\left(i\left(e_{j}\right) \beta\right), \\
& \Xi: T M \otimes \Lambda_{7}^{2} \rightarrow \Lambda^{3} T^{*} M, \quad \alpha \otimes \beta \longmapsto \alpha \wedge \beta=3 \operatorname{alt}(\alpha \otimes \beta),
\end{aligned}
$$


where $p_{7}: \Lambda^{2} T^{*} M \rightarrow \Lambda_{7}^{2} T^{*} M$ is the orthogonal projection.

Note that the map $\Xi \circ \Theta$ is symmetric and $\operatorname{Spin}(7)$-equivariant, so that its eigenspaces must be $\Lambda_{8}^{3} T^{*} M$ and $\Lambda_{48}^{3} T^{*} M$. Taking $i\left(e_{0}\right) \Omega \in \Lambda_{8}^{3} T^{*} M$ and $e^{123}+e^{145} \in \Lambda_{48}^{3} T^{*} M$ one can show that the eigenvalues are $\frac{9}{4}$ on $\Lambda_{8}^{3} T^{*} M$ and $\frac{1}{2}$ on $\Lambda_{48}^{3} T^{*} M$.

Proposition 5.1. Given a $\operatorname{Spin}(7)$ structure, there exists a unique characteristic connection whose torsion $\mathrm{T} \in \Lambda^{3} T^{*} M$ is given by:

$$
\mathrm{T}=-\delta \Omega-\frac{7}{6} *(\theta \wedge \Omega)
$$

Proof. A connection with skew-symetric torsion $\mathrm{T} \in \Lambda^{3} T^{*} M$ is given by $\nabla_{X} Y+\frac{1}{2} \mathrm{~T}(X, Y, \cdot)^{\#}$, where $\mathrm{T}(X, Y, \cdot)^{\#}$ is the vector field such that $\left(\mathrm{T}(X, Y, \cdot)^{\#}\right)^{*}=\mathrm{T}(X, Y, \cdot)$. Thus, the lift to the spinorial bundle is $\nabla_{X} \phi+\frac{1}{4} i(X) \mathrm{T} \phi$.

Since the condition $\nabla^{c} \Omega=0$ is equivalent to $\nabla^{c} \eta=0$ and the kernel of the Clifford product by $\eta$ on $\Lambda^{2} T^{*} M$ is $\Lambda_{21}^{2} T^{*} M$, the set of characteristic connections is isomorphic to the set of 3-forms $\mathrm{T} \in \Lambda^{3} T^{*} M$ such that

$$
-4 c^{-1} \nabla_{X} \eta=i(X) T \eta=p_{7}(i(X) T), \quad \forall X \in \mathfrak{X}(M) .
$$

The last equality may be rewritten as $-4 \Theta\left(c^{-1} \nabla \eta\right)=\Theta(T)$. From the definition of $\gamma_{48}$ given in Lemma 4.4 we have: $-4 \Xi\left(c^{-1} \nabla \eta\right)=-12 \operatorname{alt}\left(c^{-1} \nabla \eta\right)=\frac{1}{7}\left(\gamma_{48}-3 \gamma_{8}\right)$. Finally, taking into account the eigenvalues of $\Xi \circ \Theta$, we get:

$$
T=\frac{1}{7}\left(2 \gamma_{48}-\frac{4}{3} \gamma_{8}\right)=* d \Omega-\frac{4}{3} \gamma_{8}=-\delta \Omega-\frac{7}{6} *(\theta \wedge \Omega) .
$$

To obtain the second equality we have used the formula for $d \Omega$ from Lemma 4.4. To check the last one, note that $\gamma_{8}=i(V) \Omega=*\left(V^{*} \wedge \Omega\right)=\frac{7}{8} * \theta \wedge \Omega$.

\section{6. $\mathrm{G}_{2}$ Distributions}

In this section we define the notion of $\mathrm{G}_{2}$ distribution on a $\operatorname{Spin}(7)$ manifold in terms of spinors, and we study the torsion of the structure with respect to a suitable connection on the distribution. Then, we relate the $\operatorname{Spin}(7)$ structure of the ambient manifold with the $\mathrm{G}_{2}$ structure of the distribution. This approach enables us to study $\mathrm{G}_{2}$ structures on submanifolds of $\operatorname{Spin}(7)$ manifolds, $S^{1}$-principal fibre bundles over $G_{2}$ manifolds and warped products of manifolds admitting a $G_{2}$ structure with $\mathbb{R}$. Our analysis is very similar to the description of $G_{2}$ structures from a spinorial viewpoint, done in [1], which we briefly recall.

A 7-dimensional Riemannian manifold $(Q, g)$ can be equipped with a $\mathrm{G}_{2}$ structure if it is spin and its spinorial bundle $\Sigma(Q)$ admits a unitary section $\eta$. A cross product is then constructed from the spinor and is determined by a 3 -form $\Psi$. Denote by $\nabla^{Q}$ both the Levi-Civita connection of the manifold and its lift to the spinorial bundle; an endomorphism $\mathcal{S}$ of $T Q$ is defined by the condition:

$$
\nabla_{X}^{Q} \eta=\mathcal{S}(X) \eta \text {. }
$$

The intrinsic torsion is $-\frac{2}{3} i(\mathcal{S}) \Psi\left[1\right.$, Proposition 4.4], so that pure types of $\mathrm{G}_{2}$ structures are given by the $\mathrm{G}_{2}$ irreducible components of $\operatorname{End}(T Q)$. It is known that $\operatorname{End}\left(\mathbb{R}^{7}\right)=\chi_{1} \oplus \chi_{2} \oplus \chi_{3} \oplus \chi_{4}$, where $\chi_{i}$ are irreducible $\mathrm{G}_{2}$ representations, defined by:

$$
\chi_{1}=\langle\mathrm{Id}\rangle, \quad \chi_{2}=\mathfrak{g}_{2}, \quad \chi_{3}=\operatorname{Sym}_{0}^{2}\left(\mathbb{R}^{7}\right), \quad \chi_{4}=\left\{A: \mathbb{R}^{7} \rightarrow \mathbb{R}^{7}: A(X)=X \times S, \quad S \in \mathbb{R}^{7}\right\},
$$

where $\operatorname{Sym}_{0}^{2}\left(\mathbb{R}^{7}\right)$ denotes the set of symmetric and traceless endomorphisms. The dimensions of the previous spaces are 1, 14, 27 and 7 respectively.

If we denote by $R_{Q}$ a $\mathrm{G}_{2}$ reduction of the $\mathrm{SO}(7)$ principal bundle $P(Q)$ and define $\chi_{i}(Q)=R_{Q} \times_{\mathrm{G}_{2}} \chi_{i}$, then the pure classes of $\mathrm{G}_{2}$ structures are determined by the condition $\mathcal{S} \in \chi_{i}(Q)$. For instance, nearly parallel $\mathrm{G}_{2}$ structures verify $\mathcal{S} \in \chi_{1}(Q)$, almost parallel or calibrated are those with $\mathcal{S} \in \chi_{2}(Q)$, and locally conformally calibrated are such that $\mathcal{S} \in \chi_{4}(Q)$. Indeed in the nearly parallel case it holds that $\mathcal{S}(X)=\lambda_{0} X$ for some $\lambda_{0} \in \mathbb{R}$. Moreover mixed classes are also relevant, for instance cocalibrated structures which correspond to $\mathcal{S} \in \chi_{1}(Q) \oplus \chi_{3}(Q)$.

Taking this into account, we define $\mathrm{G}_{2}$ structures on distributions and characterise the existence of such structures.

Definition 6.1. Let $(M, g)$ be an oriented 8-dimensional Riemannian manifold and let $\mathcal{D}$ be a cooriented distribution of codimension 1 . We say that $\mathcal{D}$ has a $\mathrm{G}_{2}$ structure if the principal $\mathrm{SO}(7)$ bundle $P(\mathcal{D})$ is spin and the spinorial bundle $\Sigma(\mathcal{D})$ admits a unitary section. 
Lemma 6.2. Consider an oriented 8-dimensional Riemannian manifold $(M, g)$ and a cooriented distribution $\mathcal{D}$ of codimension 1. Take a unitary vector field $N$, perpendicular to $\mathcal{D}$ such that $T M=\langle N\rangle \oplus \mathcal{D}$ as oriented bundles. The manifold $M$ is spin if and only if the bundle $P(\mathcal{D})$ is spin. In this case, the spinorial bundles are related by $\Sigma(\mathcal{D})=\Sigma^{+}(M)$ and it holds

$$
X \cdot_{\mathcal{D}} \phi=N X \phi \text {, if } X \in \mathcal{D}, \quad \phi \in \Sigma(\mathcal{D}) \text {, }
$$

where we have suppressed the symbol $\cdot_{M}$ to denote the Clifford product on $M$.

Therefore, $M$ has a $\operatorname{Spin}(7)$ structure if and only if $\mathcal{D}$ has a $\mathrm{G}_{2}$ structure.

Proof. The bundle $P(\mathcal{D})$ is a reduction of $P(M)$, since we have the following inclusion:

$$
i: P(\mathcal{D}) \rightarrow P(M), \quad\left(X_{1}, \ldots, X_{7}\right) \rightarrow\left(N, X_{1}, \ldots, X_{7}\right) .
$$

Suppose that $P(\mathcal{D})$ is spin and denote the spin bundle by $\pi_{\mathcal{D}}: \tilde{P}(\mathcal{D}) \rightarrow P(\mathcal{D})$. Then, we can define the principal $\operatorname{Spin}(8)$ bundle $\tilde{P}(M)=\tilde{P}(\mathcal{D}) \times_{\operatorname{Spin}(7)} \operatorname{Spin}(8)$ and the map:

$$
\pi_{M}: \tilde{P}(M) \rightarrow P(M), \quad[\tilde{F}, \tilde{\varphi}] \rightarrow \operatorname{Ad}(\tilde{\varphi})\left(i\left(\pi_{\mathcal{D}}(\tilde{F})\right)\right),
$$

which is a double covering and $A d$-equivariant. Therefore, $M$ is spin. Reciprocally, if $M$ is spin then the pullback $i^{*}(\tilde{P}(M))$ is the spin bundle of $P(\mathcal{D})$.

Moreover, the irreducible 8-dimensional representation of $\mathrm{Cl}_{7}$ which maps the volume form to the identity can be constructed from the composition

$$
\mathrm{Cl}_{7} \rightarrow \mathrm{Cl}_{8}^{0} \stackrel{\rho}{\rightarrow} \mathrm{GL}\left(\Delta^{+}\right)
$$

where the first map is induced by the embedding $\mathbb{R}^{7} \rightarrow \mathrm{Cl}_{8}^{0}, v \rightarrow e_{0} v$, denoting by $\left(e_{0}, \ldots, e_{7}\right)$ the canonical basis of $\mathbb{R}^{8}$.

Therefore, the spinorial bundle $\Sigma(\mathcal{D})$ coincides with $\Sigma(M)^{+}$and the Clifford products are related by the formula (5).

From now on we assume that the manifold $(M, g)$ has a $\operatorname{Spin}(7)$ structure $\Omega$, constructed from a unitary section $\eta$ of the spinorial bundle $\Sigma(M)^{+}$, as in Definition 3. We equip $M$ with a distribution as in Lemma 6.2.

Remarks 6.3. In this situation, we have the following:

1. If $\beta \in \Lambda^{2 k} T^{*} \mathcal{D}$ and $\phi \in \Sigma(\mathcal{D})$ then $\beta \cdot \mathcal{D} \phi=\beta \phi$.

2. There is an orthogonal decomposition $\Sigma(\mathcal{D})=\langle\eta\rangle \oplus(\mathcal{D} \cdot \mathcal{D} \eta)$.

3. The section $\eta$ defines a cross product on $\mathcal{D}$ by means of:

$$
(X \times Y) \eta=\left(X^{*} \wedge Y^{*}\right) \eta=(X Y+g(X, Y)) \eta,
$$

which is determined by $\Psi_{\mathcal{D}}(X, Y, Z)=(X \eta,(Y \times Z) \eta)=-(\eta, X Y Z \eta)$.

4. The cross product is determined by $\Psi_{\mathcal{D}}=i(N) \Omega$. Therefore, using that $* \Omega=\Omega$ we get $\Omega=$ $N^{*} \wedge \Psi_{\mathcal{D}}+*_{\mathcal{D}} \Psi_{\mathcal{D}}$

We equip $\mathcal{D}$ with a suitable connection which is determined by the covariant derivative of the ambient manifold.

Definition 6.4. The covariant derivative of $\mathcal{D}$ induced by $M, \nabla^{\mathcal{D}}$, is given by the expression:

$$
\nabla_{X}^{M} Y=\nabla_{X}^{\mathcal{D}} Y+g(\mathcal{T}(X), Y) N, \quad X, Y \in \mathcal{D}
$$

where $\mathcal{T} \in \operatorname{End}(\mathcal{D})$ is given by: $2 g(\mathcal{T}(X), Y)=-N(g(X, Y))-g([X, N], Y)-g([Y, N], X)+g([X, Y], N)$.

We will decompose $\mathcal{T}$ into its symmetric and skew-symmetric parts, which we call $\mathcal{W}$ and $\mathcal{L}$ respectively. The connection $\nabla^{\mathcal{D}}$ is a metric connection and the tensor $\mathcal{L}=-\frac{1}{2} d N^{*}$ measures the lack of integrability of the distribution.

We will also denote by $\nabla^{\mathcal{D}}$ the lift of this connection to the spinorial bundle $\Sigma(\mathcal{D})$. This connection is metric with respect to $(\cdot, \cdot)$ and behaves as a derivation with respect to the Clifford product. Hence, $\nabla^{\mathcal{D}} \eta \in\langle\eta\rangle^{\perp}$, and there is an endomorphism of $\mathcal{D}$, that we will call $\mathcal{S}_{\mathcal{D}}$, such that $\nabla_{X}^{\mathcal{D}} \eta=\mathcal{S}_{\mathcal{D}}(X) \cdot \mathcal{D} \eta$. Therefore, if we define $\chi(\mathcal{D})=R_{\mathcal{D}} \times \chi_{i}$, where $R_{\mathcal{D}}$ is the $\mathrm{G}_{2}$ reduction of $P(\mathcal{D})$ determined by $\Psi_{\mathcal{D}}$, we have a splitting of $\operatorname{End}(\mathcal{D})$ and we can decompose $\mathcal{S}$ according to it:

$$
\mathcal{S}_{\mathcal{D}}(X)=\lambda \mathrm{Id}+S_{2}+S_{3}+S_{4}
$$

where $\lambda \in C^{\infty}(M), S_{2} \in \chi_{2}(\mathcal{D}), S_{3} \in \chi_{3}(\mathcal{D}), S_{4} \in \chi_{4}(\mathcal{D})$, and let $S \in \mathcal{D}$ be such that $S_{4}(X)=X \times S$. 
We can relate these components with the $\operatorname{Spin}(7)$ structure defined on $M$. First of all, since $g\left(\nabla_{X} N, Y\right)=$ $-g\left(\nabla_{X} Y, N\right)$ we get that the connection $\nabla^{M}$ at $\Sigma(M)^{+}$in the direction of $\mathcal{D}$ is given by:

$$
\nabla_{X}^{M} \eta=\nabla_{X}^{\mathcal{D}} \eta-\frac{1}{2} N \mathcal{T}(X) \eta=N \mathcal{A}(X) \eta
$$

where $\mathcal{A}=\mathcal{S}_{\mathcal{D}}-\frac{1}{2} \mathcal{T}$. We can decompose $\mathcal{L}$ and $\mathcal{W}$ according to the splitting of End $(\mathcal{D})$ into irreducible parts and then decompose $\mathcal{A}$ :

1. $\mathcal{L}=L_{2}+L_{4}$, where $L_{2} \in \chi_{2}(\mathcal{D}), L_{4} \in \chi_{4}(\mathcal{D})$ and let $L \in \mathcal{D}$ such that $L_{4}(X)=X \times L$.

2. $\mathcal{W}=h \mathrm{Id}+W_{3}$, where $h \in C^{\infty}(M), W_{3} \in \chi_{3}(\mathcal{D})$.

3. $\mathcal{A}=\mu \mathrm{Id}+A_{2}+A_{3}+A_{4}$, where $\mu=\lambda-\frac{h}{2}, A_{2}=S_{2}-\frac{1}{2} L_{2}, A_{3}=S_{3}-\frac{1}{2} W_{3}, A_{4}=S_{4}-\frac{1}{2} L_{4}$. We will also denote $A=S-\frac{1}{2} L$.

We are going to compute $* d \Omega$ in terms of the previous endomorphisms and $\nabla_{N}^{\mathcal{D}} \eta$. Our first lemma is deduced from [1, Theorems 4.6,4.8].

Lemma 6.5. If we take an oriented orthonormal local frame of $\mathcal{D},\left(X_{1}, \ldots, X_{7}\right)$ then

$$
\sum_{i=1}^{7} X_{i} \mathcal{A}\left(X_{i}\right) \eta=-7 \mu \eta-6 N A \eta
$$

Proof. We will split the endomorphism $\mathcal{A}$ into its $\mathrm{G}_{2}$ irreducible components and then compute each term separately. It is obvious that $\sum_{i=1}^{7} X_{i} \mu X_{i} \eta=-7 \mu \eta$. Moreover,

$$
\sum_{i=1}^{7} X_{i}\left(X_{i} \times A\right) \eta=\sum_{i=1}^{7} X_{i}\left(X_{i} N A-g\left(X_{i}, A\right) N\right) \eta=-6 N A .
$$

Finally consider the $\mathrm{G}_{2}$-equivariant map, $m: \mathcal{D} \otimes \mathcal{D} \rightarrow \Sigma(\mathcal{D}), m(X, Y)=X Y \eta$. By dimensional reasons, its kernel must be $\chi_{2}(\mathcal{D}) \oplus \chi_{3}(\mathcal{D})$. Therefore, if $k \in\{2,3\}$ we have that:

$$
\sum_{i=1}^{7} X_{i} A_{k}\left(X_{i}\right) \eta=m\left(\sum_{i=1}^{7}\left(A_{k}\right)_{i j} X_{i} X_{j}\right)=0
$$

where we have denoted $\left(A_{k}\right)_{i j}$ the entries of the matrix $A_{k}$ with respect to the basis $\left(X_{1}, \ldots, X_{7}\right)$.

Remarks 6.6 .

1. Since $\nabla_{N}^{M} \eta$ is perpendicular to $\eta$ we can take $U \in \mathcal{D}$ such that $\nabla_{N}^{M} \eta=-N U \eta$.

In order to compute $\nabla_{N}^{M} \eta$ we may take $F=\left(X_{0}, X_{1}, \ldots, X_{7}\right)$ a local orthonormal frame of $M$ such that $N=X_{0}$, a lifting $\tilde{F} \in \tilde{P}(M)$ and write $\eta(p)=[\tilde{F}, s(p)]$. With this notation we have:

$$
\begin{aligned}
\nabla_{X_{0}}^{M} \eta & =\left[\tilde{F}, d s\left(X_{0}\right)\right]+\frac{1}{2} \sum_{0 \leq i<j \leq 7} g\left(\nabla_{X_{0}} X_{i}, X_{j}\right) X_{i} X_{j} \eta \\
& =\left[\tilde{F}, d s\left(X_{0}\right)\right]+\frac{1}{2}\left(X_{0} \nabla_{X_{0}} X_{0}+\sum_{1 \leq i<j \leq 7} g\left(\nabla_{X_{0}} X_{i}, X_{j}\right) X_{i} X_{j}\right) \eta
\end{aligned}
$$

Then, $U$ depends on the local information of the section and $\nabla_{X_{0}} X_{i}$.

2. The Dirac operator of $M$ is

$$
D^{M} \eta=U \eta+\sum_{i=1}^{7} X_{i} N \mathcal{A}\left(X_{i}\right) \eta=(U-6 A+7 \mu N) \eta .
$$

Lemma 6.7. If we define the forms on $\mathcal{D}, \beta_{2} \in \Lambda^{2} \mathcal{D}^{*}$ and $\beta_{3} \in \Lambda^{3} \mathcal{D}^{*}$ by:

$$
\beta_{2}(X, Y)=g\left(A_{2}(X), Y\right), \quad \beta_{3}(X, Y, Z)=\operatorname{alt}\left(i\left(A_{3}\right)(\cdot) \Psi_{\mathcal{D}}\right)(X, Y, Z),
$$

then:

1. $N^{*} \wedge i(N)\left(12 \operatorname{alt}\left(c^{-1} \nabla \eta\right)\right)=i(U-2 A)\left(N^{*} \wedge \Psi_{\mathcal{D}}\right)-2 N^{*} \wedge \beta_{2}$,

2. $\left.12 \operatorname{alt}\left(c^{-1} \nabla \eta\right)\right|_{Q}=\left.3 i(\mu N-A) \Omega\right|_{Q}+3 \beta_{3}$. 
Proof. The first equality is a consequence of the symmetric or skew-symmetric properties of each factor:

$$
\begin{aligned}
12 \operatorname{alt}\left(c^{-1} \nabla \eta\right)(N, X, Y) & =-(X Y \eta, N U \eta)-(N Y \eta, N \mathcal{A}(X) \eta)+(N Y \eta, N \mathcal{A}(X) \eta) \\
& =-i(U) \Psi_{\mathcal{D}}(X, Y)-2\left(Y \eta,\left(A_{2}(X)+X \times A\right) \eta\right) \\
& =\left(i(U-2 A)\left(N^{*} \wedge \Psi_{\mathcal{D}}\right)-2 N^{*} \wedge \beta_{2}\right)(N, X, Y) .
\end{aligned}
$$

To check the second one, note that $\left.12 \operatorname{alt}\left(c^{-1} \nabla \eta\right)\right|_{Q}=3 \operatorname{alt}\left(i(\mathcal{A}(\cdot)) \Psi_{\mathcal{D}}\right)$. We compute separatedly each term in the decomposition of $\mathcal{A}$.

It is evident that $3 \operatorname{alt}\left(i(\mu \mathrm{Id}) \Psi_{\mathcal{D}}\right)(X, Y, Z)=3 \mu \Psi_{\mathcal{D}}(X, Y, Z)$ and $3 \operatorname{alt}\left(i\left(A_{3}(\cdot)\right) \Psi_{\mathcal{D}}\right)=3 \beta_{3}$. Moreover, we have that $\operatorname{alt}\left(i\left(A_{2}(\cdot)\right) \Psi_{\mathcal{D}}\right)=0$ because $A_{2} \in \chi_{2}(Q)$. Finally, if $X, Y$ and $Z$ are orthonormal vectors in $T Q$, then:

$$
i\left(A_{4}(X)\right) \Psi_{\mathcal{D}}(Y, Z)=(X \times A \eta, Y \times Z \eta)=(X A \eta, Y Z \eta)=-(A \eta,(X \times Y \times Z) \eta) .
$$

Therefore, $3 \operatorname{alt}\left(i\left(A_{4}(\cdot)\right) \Psi_{\mathcal{D}}\right)(X, Y, Z)=-3(A \eta, X \times Y \times Z \eta)$.

From lemmas 6.5 and 6.7 and the decomposition of $* d \Omega$ obtained in Proposition 4.4 we conclude:

Proposition 6.8. Let $U \in \mathcal{D}$ such that $\nabla_{N}^{M} \eta=-N U \eta$ and define the forms on $\mathcal{D}, \beta_{2} \in \Lambda^{2} \mathcal{D}^{*}$ and $\beta_{3} \in \Lambda^{3} \mathcal{D}^{*}$ by:

$$
\beta_{2}(X, Y)=g\left(A_{2}(X), Y\right), \quad \beta_{3}(X, Y, Z)=\operatorname{alt}\left(i\left(A_{3}\right)(\cdot) \Psi_{\mathcal{D}}\right)(X, Y, Z) .
$$

Then, the pure components of $* d \Omega$ in terms of the $\mathrm{G}_{2}$ structure are:

$$
\begin{aligned}
(* d \Omega)_{48} & =\frac{2}{7}\left(-4 i(A+U) N^{*} \wedge \Psi+3 i(A+U) *_{\mathcal{D}} \Psi_{\mathcal{D}}\right)+4 N^{*} \wedge \beta_{2}-6 \beta_{3}, \\
(* d \Omega)_{8} & =\frac{8}{7} i(U-6 A+7 \mu N)\left(N^{*} \wedge \Psi_{\mathcal{D}}+*_{\mathcal{D}} \Psi_{\mathcal{D}}\right) .
\end{aligned}
$$

6.1. Hypersurfaces. Consider an 8-dimensional $\operatorname{Spin}(7)$ manifold $(M, g)$, whose $\operatorname{Spin}(7)$ form is constructed from a unitary section $\eta$ of the spinorial bundle $\Sigma(M)^{+}$, as in Definition 3. Let $Q$ be an oriented hypersurface and take a unitary vector field $N$ such that $T M=\langle N\rangle \oplus T Q$ as oriented vector bundles.

The tubular neighbourhood theorem guarantees the existence of a cooriented distribution $\mathcal{D}$ defined on a neighbourhood $O$ of $Q$ such that $\left.\mathcal{D}\right|_{Q}=T Q$. The coorientation is determined by a unitary extension of the normal vector field that we also denote by $N$. Both $\mathcal{D}$ and $Q$ have $\mathrm{G}_{2}$ structures determined by the spinor $\eta$; we are going to relate them using Proposition 6.8 in the manifold $O$.

Note that the Levi-Civita connection of the hypersurface $Q$ is $\left.\nabla^{\mathcal{D}}\right|_{Q}$. Moreover, $\left.\mathcal{L}\right|_{Q}=0$ and $\left.\mathcal{W}\right|_{Q}$ is the Weingarten operator. Therefore, the restriction of $\mathcal{S}_{\mathcal{D}}$ at $Q$ is the endomorphism $\mathcal{S}$ of the submanifold $Q$. Decompose $\left.\mathcal{S}\right|_{Q}$ and $\left.\mathcal{W}\right|_{Q}$ with respect of the $\mathrm{G}_{2}$ splitting of $\operatorname{End}(T Q)$ :

1. $\mathcal{S}=\lambda \mathrm{Id}+S_{2}+S_{3}+S_{4}$

2. $\left.\mathcal{W}\right|_{Q}=7 H \mathrm{Id}+W_{3}$,

where $\lambda \in C^{\infty}(M), S_{2} \in \chi_{2}(Q), S_{3}, W_{3} \in \chi_{3}(Q), S_{4} \in \chi_{4}(Q)$ and $H \in C^{\infty}(Q)$ is the mean curvature. We will also denote by $S$ the vector in $T Q$ such that $S_{4}(X)=X \times S$.

Corollary 6.9. Let $U \in T Q$ such that $\left.\nabla_{N}^{M} \eta\right|_{Q}=-N U \eta$ and $\Psi_{Q}=i(N) \Omega$. Define the forms on $Q$, $\beta_{2} \in \Lambda^{2} T^{*} Q$ and $\beta_{3} \in \Lambda^{3} T^{*} Q$ by:

$$
\beta_{2}(X, Y)=g\left(S_{2}(X), Y\right), \quad \beta_{3}(X, Y, Z)=\operatorname{alt}\left(i\left(\left(S_{3}-\frac{1}{2} W_{3}\right)(\cdot)\right) \Psi_{\mathcal{D}}\right)(X, Y, Z) .
$$

Then, the pure components of $* d \Omega$ in terms of the $\mathrm{G}_{2}$ structure are:

$$
\begin{aligned}
(* d \Omega)_{48} & =\frac{2}{7}\left(-4 i(S+U) N^{*} \wedge \Psi_{Q}+3 i(S+U) *_{Q} \Psi_{Q}\right)+4 N^{*} \wedge i^{*} \beta_{2}-6 \beta_{3}, \\
(* d \Omega)_{8} & =\frac{8}{7} i\left(U-6 S+7\left(\lambda-\frac{7}{2} H\right) N\right)\left(N^{*} \wedge \Psi_{Q}+*_{Q} \Psi_{Q}\right) .
\end{aligned}
$$

Remark 6.10. Note that the condition $\left.\nabla_{N} \eta\right|_{Q}=-N U \eta$ does not depend on the extension of the vectors. Moreover, we can compute $U$ taking into account equation (6). The terms involved are extrinsic and not encoded in $\mathcal{S}$ and $\mathcal{W}$.

Therefore, the $\operatorname{Spin}(7)$ type of the ambient manifold provides relations between the $\mathrm{G}_{2}$ type of the hypersurface, the vector $U$ and the Weingarten operator. Before stating the result, we recall that a hypersurface is said to be totally geodesic if $\mathcal{W}=0$, totally umbilic if $W_{3}=0$ and minimal if $H=0$. 
Theorem 6.11. Let $(M, g)$ be a Riemannian manifold endowed with a $\operatorname{Spin}(7)$ structure determined by a spinor $\eta$. Let $Q$ be an oriented hypersurface with normal vector $N$ and let $U \in T Q$ be such that $\left.\nabla_{N} \eta\right|_{Q}=-N U \eta$.

1. If $M$ has a parallel Spin(7) structure, then $Q$ has a cocalibrated $\mathrm{G}_{2}$ structure. Moreover,

$1.1 \mathcal{S}=0$ if and only if $Q$ is totally geodesic.

$1.2 \mathcal{S} \in \chi_{1}(Q)$ if and only if $Q$ is totally umbilic.

$1.3 \mathcal{S} \in \chi_{3}(Q)$ if and only if $Q$ is a minimal hypersurface.

2. If $M$ has a locally conformally parallel Spin(7) structure, then $\mathcal{S} \in \chi_{1}(Q) \oplus \oplus \chi_{3}(Q) \oplus \chi_{4}(Q)$. Indeed,

$2.1 \mathcal{S} \in \chi_{1}(Q)$ if and only if $U=0$ and $Q$ is totally umbilic.

$2.2 \mathcal{S} \in \chi_{1}(Q) \oplus \chi_{4}(Q)$ if and only if $Q$ is totally umbilic.

3. If $M$ has a balanced $\operatorname{Spin}(7)$ structure, then:

$3.1 \mathcal{S} \in \chi_{2}(Q) \oplus \chi_{3}(Q)$ if and only if $U=0$ and $Q$ is a minimal hypersurface.

$3.2 \mathcal{S} \in \chi_{1}(Q) \oplus \chi_{2}(Q) \oplus \chi_{3}(Q)$ if and only if $U=0$.

$3.3 \mathcal{S} \in \chi_{2}(Q) \oplus \chi_{3}(Q) \oplus \chi_{4}(Q)$ if and only if $Q$ is a minimal hypersurface.

Proof. The parallel case follows from the equalities $U=S=0, S_{2}=0,2 \lambda=7 \mathrm{H}$ and $2 S_{3}=W_{3}$. The locally conformally parallel case follows from the equalities $U=-S, S_{2}=0$ and $2 S_{3}=W_{3}$, which imply that $S \in \chi_{1}(Q) \oplus \chi_{2}(Q) \oplus \chi_{3}(Q)$. Finally the balanced case follows from $U=6 S$ and $2 \lambda=7 H$.

6.2. Principal bundles over a $\mathrm{G}_{2}$ manifold. Let $Q$ be a $\mathrm{G}_{2}$ manifold and let $\pi: M \rightarrow Q$ be a $G=\mathbb{R}$ or $G=S^{1}$ principal bundle over $Q$; identify its Lie algebra $\mathfrak{g}$ with $\mathbb{R}$.

Define the vertical field $N(p)=\left.\frac{d}{d t}\right|_{t=0}(p \exp (t))$. A connection $\omega: T M \rightarrow \mathfrak{g}$ defines a horizontal distribution $\mathcal{H}$ and we can define a metric on $M$ such that:

1. The map $d \pi: \mathcal{H}_{p} \rightarrow T_{\pi(p)} Q$ is an isometry

2. The vector $N(p)$ is unitary and perpendicular to $\mathcal{H}_{p}$.

The projection $d \pi$ induces a map $p: P(\mathcal{H}) \rightarrow P(Q)$ so that the pullback to $\tilde{P}(Q)$ defines a spin structure $\tilde{P}(\mathcal{H})$ on $P(\mathcal{H})$. The map $\tilde{p}: \tilde{P}(\mathcal{H}) \rightarrow \tilde{P}(Q)$, which is canonically defined, has the property that $\tilde{p}(\tilde{\varphi} \tilde{F})=\tilde{\varphi} \tilde{p}(\tilde{F})$ if $\tilde{\varphi} \in \operatorname{Spin}(8)$, inducing therefore a map between the spinorial bundles, which we call $\bar{p}$. Note that this map gives isomorphisms $\Sigma(\mathcal{H})_{p} \rightarrow \Sigma(Q)_{\pi(p)}$. Moreover, let $X \in T Q$ and denote by $X^{h}$ its horizontal lift, then $\bar{p}\left(X^{h} \cdot \mathcal{H} \phi\right)=X \bar{p}(\phi)$. Therefore, a section $\bar{\eta}: Q \rightarrow \Sigma(Q)$ allows us to define a section $\eta: M \rightarrow \Sigma(\mathcal{H})$ by means of the expression $\bar{p}(\eta)=\bar{\eta}$. If we denote by $\Psi_{Q}$ the $\mathrm{G}_{2}$ form on $Q$, then $\Psi_{\mathcal{D}}=\pi^{*} \Psi_{Q}$.

Furthermore, one can check that $\nabla_{X^{h}}^{\mathcal{H}} Y^{h}=\left(\nabla_{X}^{Q} Y\right)^{h}$. Hence, if we take $\mathcal{S} \in \operatorname{End}(Q)$ such that $\nabla_{X}^{Q} \bar{\eta}=\mathcal{S}(X) \eta$, we get that the endomorphism of the distribution $\mathcal{S}_{\mathcal{D}}$ is the lifting of $\mathcal{S}$, that is:

$$
\nabla_{X^{h}}^{\mathcal{H}} \eta=\mathcal{S}(X)^{h} \eta
$$

Therefore the distribution $\mathcal{H}$ and the manifold $Q$ have the same type of $\mathrm{G}_{2}$ structure. In order to classify the $\operatorname{Spin}(7)$ structure on $M$, denote the curvature of the connection $\omega$ by:

$$
\mathfrak{L}(X, Y)=\left[X^{h}, Y^{h}\right]-[X, Y]^{h} \in\langle N\rangle, \quad X, Y \in T Q .
$$

Since $\mathfrak{L}(X, Y) \in\langle N\rangle$ we also denote by $\mathfrak{L}$ the 2 -form that we obtain contracting the tensor with the metric. As a skew-symmetric endomorphism, we can decompose $\mathfrak{L}=\bar{L}_{2}+\bar{L}_{4}$ where $\bar{L}_{4}(X)=X \times \bar{L}$ for some $\bar{L}$ in $T Q$.

Corollary 6.12. Suppose that $\nabla_{X}^{Q} \bar{\eta}=\mathcal{S}(X) \cdot Q \bar{\eta}$ with $\mathcal{S}(X)=\lambda \mathrm{Id}+S_{2}+S_{3}+S_{4}$ where $\lambda \in C^{\infty}(Q)$, $S_{2} \in \chi_{2}(Q), S_{3} \in \chi_{3}(Q), S_{4} \in \chi_{4}(Q)$ and let $S \in T Q$ be such that $S_{4}(X)=X \times S$. Define $\beta_{2} \in \Lambda^{2} T^{*} Q$ and $\beta_{3} \in \Lambda^{3} T^{*} Q$ by:

$$
\beta_{2}(X, Y)=g\left(S_{2}(X)-\frac{1}{4} \bar{L}_{2}(X), Y\right), \quad \beta_{3}(X, Y, Z)=\operatorname{alt}\left(i\left(S_{3}(\cdot)\right) \Psi_{Q}\right)(X, Y, Z) .
$$

The pure components of $* d \Omega$ in terms of the $\mathrm{G}_{2}$ structure are:

$$
\begin{aligned}
(* d \Omega)_{48} & =\frac{2}{7}\left(-4 i\left(S^{h}+\frac{1}{2} \bar{L}^{h}\right) N^{*} \wedge \pi^{*} \Psi_{Q}+3 i\left(S^{h}+\frac{1}{2} \bar{L}^{h}\right) \pi^{*}\left(*_{Q} \Psi_{Q}\right)\right)-4 N^{*} \wedge \pi^{*} \beta_{2}+6 \pi^{*} \beta_{3}, \\
(* d \Omega)_{8} & =\frac{8}{7} i\left(\frac{15}{4} \bar{L}^{h}-6 S^{h}+7 \lambda N\right)\left(N^{*} \wedge \pi^{*} \Psi_{Q}+\pi^{*}\left(*_{Q} \Psi_{Q}\right)\right) .
\end{aligned}
$$


Proof. The result follows immediately from Proposition 6.8 once we check that $\mathcal{W}=0, g(\mathcal{L}(X), Y)=$ $\frac{1}{2} \pi^{*} \mathfrak{L}(X, Y)$, and $U=\frac{3}{4} \bar{L}^{h}$.

First of all, since the connection $\omega$ is left-invariant we have that $\left[X^{h}, N\right]=0$ if $X \in T Q$. Thus, $\mathcal{W}=0$. Moreover, $\mathcal{L}\left(X^{h}\right)\left(Y^{h}\right)=\frac{1}{2} \mathfrak{L}(X, Y)$. Furthermore, let $F=\left(X_{1}, \ldots, X_{7}\right)$ be a local frame of $\mathcal{H}$ which lifts some local frame of $T Q$. Take a lift $\tilde{F} \in \tilde{P}(\mathcal{H})$ and write $\eta(p)=[\tilde{F}, s(p)]$. We denote $X_{0}=N$ and compute $U$ using the formula $(6)$.

By definition, if $\bar{\eta}(\pi(p))=[\tilde{p}(\tilde{F}(p)), \bar{s}(\pi(p))]$ then $s(p)=\bar{s}(\pi(p))$ so that $d s_{p}(N)=0$. Besides, according to Koszul formulas we have:

$$
\begin{aligned}
\nabla_{N} N & =0 \\
g\left(\nabla_{N} X_{i}, X_{j}\right) & =-\frac{1}{2} g\left(\left[X_{i}, X_{j}\right], N\right)=-\frac{1}{2} g\left(N, \mathfrak{L}\left(d \pi\left(X_{i}\right), d \pi\left(X_{j}\right)\right)\right) .
\end{aligned}
$$

Therefore, if we define $\gamma_{i}(X, Y)=g\left(\bar{L}_{i}(X), Y\right)$, for $i \in\{2,4\}$, then:

$$
\nabla_{N} \eta=-\frac{1}{4} \pi^{*} \mathfrak{L} \eta=-\frac{1}{4} \pi^{*} \gamma_{4} \eta=-\frac{3}{4} N \bar{L}^{h} \eta
$$

where we have used that $\pi^{*} \gamma_{2} \eta=0$ because $\mathfrak{g}_{2} \subset \mathfrak{s p i n}(7)=\Lambda_{14}^{2} \mathbb{R}^{8}$ and $\pi^{*} \gamma_{4}=-i(N) i\left(\bar{L}^{h}\right) \Omega$ so that $\pi^{*} \gamma_{4} \eta=3 N \bar{L}^{h} \eta$, as we noted in the proof of Lemma 3.1 .

6.3. Warped products. We analyze $\operatorname{Spin}(7)$ structures on warped products of a $\mathrm{G}_{2}$ manifold with $\mathbb{R}$. Recall that a warped product of two Riemannian manifolds $\left(X_{1}, g_{1}\right)$ and $\left(X_{2}, g_{2}\right)$ is $\left(X_{1} \times X_{2}, g_{1}+f_{1} g_{2}\right)$ where $f_{1}: X_{1} \rightarrow \mathbb{R}$ is a smooth function. Therefore, we have to distinguish two cases.

6.3.1. Consider a $\mathrm{G}_{2}$ manifold $(Q, g)$ and a smooth function $f: \mathbb{R} \rightarrow \mathbb{R}$. Define the Riemannian manifold $\left(M=Q \times \mathbb{R}, e^{2 f} g+d t^{2}\right)$. This is the so-called spin cone.

The distribution $\mathcal{D}=T Q$ obviously admits a $\mathrm{G}_{2}$ structure. The spinorial bundle is given by $\Sigma(M)^{+}=$ $\Sigma(T Q \times \mathbb{R})=\Sigma(Q) \times \mathbb{R}$ and Clifford products are related by $\left(X \cdot_{Q} \phi, t\right)=e^{-f} X \cdot \mathcal{D}(\phi, t)=e^{-f} \frac{\partial}{\partial t} X(\phi, t)$ if $X \in T Q$. In the last expression, we have suppressed the symbol · to denote the Clifford product on $M$.

A unitary section $\eta$ is constructed from a section $\bar{\eta}: Q \rightarrow \Sigma(Q)$ by defining $\eta: M \rightarrow \Sigma(\mathcal{D}), \eta(x, t)=$ $(\bar{\eta}(x), t)$. If we denote by $\Psi_{Q}$ the $\mathrm{G}_{2}$ form on $Q$, then $\Psi_{\mathcal{D}}=e^{3 f} \pi^{*} \Psi_{Q}$ and $*_{\mathcal{D}}\left(\Psi_{\mathcal{D}}\right)=e^{4 f} *_{Q}\left(\Psi_{Q}\right)$. In addition, since $\nabla_{X}^{\mathcal{D}} Y=\nabla_{X}^{Q} Y$ when $X, Y \in T Q$, we have that $\nabla_{X}^{\mathcal{D}} \eta=e^{-f} \mathcal{S}(X) \cdot \mathcal{D} \eta$, if $X \in T Q$ and $\nabla_{X}^{Q} \bar{\eta}=\mathcal{S}(X) \bar{\eta}$. That is, $\mathcal{S}_{\mathcal{D}}=e^{-f} \mathcal{S}$.

Corollary 6.13. Suppose that $\nabla_{X}^{Q} \bar{\eta}=\mathcal{S}(X) \cdot Q \bar{\eta}$ with $\mathcal{S}(X)=\lambda \mathrm{Id}+S_{2}+S_{3}+S_{4}$ where $\lambda \in C^{\infty}(Q)$, $S_{2} \in \chi_{2}(Q), S_{3} \in \chi_{3}(Q), S_{4} \in \chi_{4}(Q)$. Let $S \in T Q$ be such that $S_{4}(X)=X \times S$. Denote by $\Psi_{Q}$ the $\mathrm{G}_{2}$-form on $Q$ and define $\beta_{2} \in \Lambda^{2} T^{*} Q$ and $\beta_{3} \in \Lambda^{3} T^{*} Q$ by:

$$
\beta_{2}(X, Y)=g\left(S_{2}(X), Y\right), \quad \beta_{3}(X, Y, Z)=\operatorname{alt}\left(i\left(S_{3}(\cdot)\right) \Psi_{Q}\right)(X, Y, Z) .
$$

The pure components of $* d \Omega$ in terms of the $\mathrm{G}_{2}$ structure are:

$$
\begin{aligned}
(* d \Omega)_{48} & =\frac{2}{7}\left(-4 e^{2 f} i(S) d t \wedge \pi^{*} \Psi_{Q}+3 e^{3 f} i(S) \pi^{*}\left(*_{Q} \Psi_{Q}\right)\right)+4 e^{f} d t^{*} \wedge \pi^{*} \beta_{2}-6 e^{2 f} \pi^{*} \beta_{3}, \\
(* d \Omega)_{8} & =\frac{8}{7} i\left(-6 e^{-f} S+7\left(\lambda e^{-f}+\frac{1}{2} f^{\prime}\right) \frac{\partial}{\partial t}\right)\left(e^{3 f} d t \wedge \pi^{*} \Psi_{Q}+e^{4 f} \pi^{*}\left(*_{Q} \Psi_{Q}\right)\right) .
\end{aligned}
$$

Proof. The result follows immediately from Proposition 6.8 once we check that $\mathcal{W}=-f^{\prime} I d, \mathcal{L}=0$ and $U=0$.

Since the distribution $\mathcal{D}$ is integrable, we have that $\mathcal{L}=0$. Take an orthonormal frame of $T Q$, $\left(X_{1}, \ldots, X_{7}\right)$ and note that $\mathcal{W}\left(X_{i}, X_{j}\right)=-f^{\prime} e^{2 f} \delta_{i j}$ so that $\mathcal{W}=-f^{\prime}$. Moreover, using the Koszul formulas we get:

$$
\nabla_{\frac{\partial}{\partial t}} \frac{\partial}{\partial t}=0=\nabla_{\frac{\partial}{\partial t}}\left(e^{-f} X_{i}\right)
$$

Therefore, using formula (6) we conclude that $\nabla_{\frac{\partial}{\partial t}} \eta=0$. 
6.3.2. Consider a $\mathrm{G}_{2}$ manifold $(Q, g)$ and a smooth function $f: Q \rightarrow \mathbb{R}$. Define the Riemannian manifold $\left(M=Q \times \mathbb{R}, g+e^{2 f} d t^{2}\right)$.

The distribution $\mathcal{D}=T Q$ obviously admits a $\mathrm{G}_{2}$ structure. The spinorial bundle is given by $\Sigma(M)^{+}=$ $\Sigma(T Q \times \mathbb{R})=\Sigma(Q) \times \mathbb{R}$ and the Clifford products are related by $\left(X \cdot{ }_{Q} \phi, t\right)=X \cdot \mathcal{D}(\phi, t)=e^{-f} \frac{\partial}{\partial t} X(\phi, t)$ if $X \in T Q$. We have suppressed again the symbol · to denote the Clifford product on $M$.

A unitary section $\eta$ is constructed from a section $\bar{\eta}: Q \rightarrow \Sigma(Q)$ by defining $\eta: M \rightarrow \Sigma(\mathcal{D}), \eta(x, t)=$ $(\bar{\eta}(x), t)$. If we denote by $\Psi_{Q}$ the $\mathrm{G}_{2}$ form on $Q$, then $\Psi_{\mathcal{D}}=\pi^{*} \Psi_{Q}$ and $*_{\mathcal{D}}\left(\Psi_{\mathcal{D}}\right)=*_{Q}\left(\Psi_{Q}\right)$. In addition, since $\nabla_{X}^{\mathcal{D}} Y=\nabla_{X}^{Q} Y$ when $X, Y \in T Q$, if we take $\mathcal{S} \in \operatorname{End}(T Q)$ with $\nabla_{X}^{Q} \bar{\eta}=\mathcal{S}(X) \bar{\eta}$, then $\mathcal{S}_{\mathcal{D}}=\mathcal{S}$.

Corollary 6.14. Suppose that $\nabla_{X}^{Q} \bar{\eta}=\mathcal{S}(X) \cdot Q \bar{\eta}$ with $\mathcal{S}(X)=\lambda \mathrm{Id}+S_{2}+S_{3}+S_{4}$ where $\lambda \in C^{\infty}(Q)$, $S_{2} \in \chi_{2}(Q), S_{3} \in \chi_{3}(Q), S_{4} \in \chi_{4}(Q)$. Let $S \in T Q$ be such that $S_{4}(X)=X \times S$. Denote by $\Psi_{Q}$ the $\mathrm{G}_{2}$-form on $Q$ and define $\beta_{2} \in \Lambda^{2} T^{*} Q$ and $\beta_{3} \in \Lambda^{3} T^{*} Q$ by:

$$
\beta_{2}(X, Y)=g\left(S_{2}(X), Y\right), \quad \beta_{3}(X, Y, Z)=\operatorname{alt}\left(i\left(S_{3}(\cdot)\right) \Psi_{Q}\right)(X, Y, Z) .
$$

The pure components of $* d \Omega$ in terms of the $\mathrm{G}_{2}$ structure are:

$$
\begin{aligned}
(* d \Omega)_{48} & =\frac{2}{7}\left(-4 i\left(S+\frac{1}{2} \operatorname{grad}(f)\right) e^{f} d t \wedge \pi^{*} \Psi_{Q}+3 i(S+\operatorname{grad}(f)) \pi^{*}\left(*_{Q} \Psi_{Q}\right)\right)+4 e^{f} d t \wedge \pi^{*} \beta_{2}-6 \pi^{*} \beta_{3}, \\
(* d \Omega)_{8} & =\frac{8}{7} i\left(\frac{1}{2} \operatorname{grad}(f)-6 S+7 \lambda e^{-f} \frac{\partial}{\partial t}\right)\left(e^{f} d t \wedge \pi^{*} \Psi_{Q}+\pi^{*}\left(*_{Q} \Psi_{Q}\right)\right) .
\end{aligned}
$$

Proof. The result follows immediatly from Proposition 6.8 once we check that $\mathcal{W}=0, \mathcal{L}=0$ and $U=\frac{1}{2} \operatorname{grad}(f)$.

Since the distribution $\mathcal{D}$ is integrable, we have that $\mathcal{L}=0$. Take an orthonormal frame of $T Q$, $\left(X_{1}, \ldots, X_{7}\right)$ and note that $\mathcal{W}\left(X_{i}, X_{j}\right)=0$. Moreover, using the Koszul formulas we get:

$$
\begin{aligned}
g\left(\nabla_{e^{-f} \frac{\partial}{\partial t}} X_{i}, X_{j}\right) & =0, \\
g\left(\nabla_{e^{-f} \frac{\partial}{\partial t}} e^{-f} \frac{\partial}{\partial t}, X_{i}\right) & =-X_{i}(f) .
\end{aligned}
$$

Therefore, using formula (6) we conclude that $\nabla_{N} \eta=-\frac{1}{2} e^{-f}\left(\frac{\partial}{\partial t}\right) \operatorname{grad}(f) \eta$.

\section{Spin(7) STRUCtures on QUASI ABELIAN LIE ALGEBRAS}

As an application of the previous section, we are going to study $\operatorname{Spin}(7)$ structures on quasi abelian Lie algebras. The geometric setting will be that of a simply connected Lie group with an invariant Spin(7) structure, endowed with an integrable distribution which inherits a $G_{2}$ structure. The integral submanifolds of the distribution are actually flat, so that the $G_{2}$ distribution will be parallel, but they will have non-trivial Weingarten operators. In some cases, finding a lattice in the Lie group will allow us to give compact examples.

First of all, let us recall the following definition:

Definition 7.1. A Lie algebra $\mathfrak{g}$ is called quasi abelian if it contains a codimension 1 abelian ideal $\mathfrak{h}$.

The information of $\mathfrak{g}$ is then encoded in $a d(x)$ for any vector $x$ transversal to $\mathfrak{h}$. The following result shows that $\mathfrak{h}$ is unique in $\mathfrak{g}$ with exception of the Lie algebras $\mathbb{R}^{n}$ and $L_{3} \oplus \mathbb{R}^{n-3}$, where $L_{3}$ is the Lie algebra of the 3 -dimensional Heisenberg group, which is generated by $x, y, z$ with relations $[x, y]=z$ and $[x, z]=[y, z]=0$.

Lemma 7.2. Let $\mathfrak{g}$ be a $n$-dimensional quasi abelian Lie algebra with $n \geq 3$. If $\mathfrak{g}$ is not isomorphic to $\mathbb{R}^{n}$ or $L_{3} \oplus \mathbb{R}^{n-3}$, then it has a unique codimension 1 abelian ideal. Moreover, codimension 1 abelian ideals on $L_{3} \oplus \mathbb{R}^{n-3}$ are parametrized by $\mathbb{R P}^{1}$.

Proof. Suppose that $\mathfrak{g}$ is not isomorphic to $\mathbb{R}^{n}$ and let $\mathfrak{h}$ be a codimension 1 abelian ideal with a transversal vector $x$. Let $\mathfrak{h}^{\prime}$ be a codimension 1 abelian ideal different from $\mathfrak{h}$. If $u \in \mathfrak{h}$ is such that $x+u \in \mathfrak{h}^{\prime}$ and $v \in \mathfrak{h} \cap \mathfrak{h}^{\prime}$, then $0=[x+u, v]=\operatorname{ad}(x)(v)$. Since $\mathfrak{h} \cap \mathfrak{h}^{\prime}$ is $(n-2)$-dimensional and $\mathfrak{g}$ is not abelian we conclude that $\mathfrak{h} \cap \mathfrak{h}^{\prime}=\operatorname{ker}\left(\left.\operatorname{ad}(x)\right|_{\mathfrak{h}}\right)$ and $\operatorname{ad}(x)(\mathfrak{h})=\langle z\rangle$ for some $z \in \mathfrak{h}$. Take $y \in \mathfrak{h}$ with $[x, y]=z$ and observe that $z \in[\mathfrak{g}, \mathfrak{g}] \subset \mathfrak{h}^{\prime}$, that is, $z \in \mathfrak{h} \cap \mathfrak{h}^{\prime}$ and $[x, z]=0$. Therefore, $\mathfrak{g}$ is isomorphic to $L_{3} \oplus \mathbb{R}^{n-3}$.

Moreover, from the discussion above we get that $\mathfrak{h}^{\prime}=\langle v, z\rangle \oplus \mathbb{R}^{5}$ for some $v \in\langle x, y\rangle$. Conversely, all the subspaces of the previous form are actually codimension 1 abelian ideals. Therefore, they are parametrized by $\mathbb{R P}^{1}$. 
An invariant $\operatorname{Spin}(7)$ structure on a Lie group is determined by the choice of a $\operatorname{Spin}(7)$ form $\Omega$, which is in turn determined by a direction of the spinorial space $\Delta^{+}$.

Define the set $\mathcal{Q} \mathcal{A}$ with elements $\left(\mathfrak{g}, \mathfrak{h}, g, \nu_{g}, \Omega\right)$ where $\mathfrak{g}$ is a non-trivial quasi abelian Lie algebra with a marked codimension 1 abelian ideal $\mathfrak{h}, g$ is a metric on $\mathfrak{g}, \nu_{g}$ is a volume form on $\mathfrak{g}$ and $\Omega$ is a $\operatorname{Spin}(7)$ structure on $\left(\mathfrak{g}, g, \nu_{g}\right)$. We will say that $\varphi^{\prime}:\left(\mathfrak{g}, \mathfrak{h}, g, \nu_{g}, \Omega\right) \rightarrow\left(\mathfrak{g}^{\prime}, \mathfrak{h}^{\prime}, g^{\prime}, \nu_{g^{\prime}}, \Omega^{\prime}\right)$ is an isomorphism if $\varphi$ is an isomorphism of Lie algebras such that $\varphi^{\prime}(\mathfrak{h})=\mathfrak{h}^{\prime},\left(\varphi^{\prime}\right)^{*} g^{\prime}=g, \varphi^{*} \nu_{g^{\prime}}=\nu_{g}$ and $\varphi^{*} \Omega^{\prime}=\Omega$.

Lemma 7.3. The set $\overline{\mathcal{Q} \mathcal{A}}$ of isomorphisms classes of $\mathcal{Q} \mathcal{A}$ is given by:

$$
\overline{\mathcal{Q} \mathcal{A}}=\left(\left(\operatorname{End}\left(\mathbb{R}^{7}\right)-\{0\}\right) \times \mathbb{P}\left(\Delta^{+}\right)\right) / \mathrm{O}(7),
$$

where $\mathrm{O}(7)$ acts via

$$
\varphi \cdot(\mathcal{E},[\eta])=\left(\operatorname{det}(\varphi) \varphi \circ \mathcal{E} \circ \varphi^{-1},[\rho(\tilde{\varphi}) \eta]\right)
$$

where $\tilde{\varphi}$ is a lifting to $\operatorname{Spin}(8)$ of the unique $\varphi^{\prime} \in \mathrm{SO}(8)$ such that $\left.\varphi^{\prime}\right|_{\mathbb{R}^{7}}=\varphi$.

Proof. A map $\left(\operatorname{End}\left(\mathbb{R}^{7}\right)-\{0\}\right) \times \mathbb{P}\left(\Delta^{+}\right) \rightarrow \mathcal{Q} \mathcal{A}$ can be defined as follows. Take $(\mathcal{E}, \bar{\eta})$ and define the Lie structure on $\mathbb{R}^{8}$ with oriented basis $\left(e_{0}, \ldots, e_{7}\right)$ such that $\mathbb{R}^{7}=\left\langle e_{1}, \ldots, e_{7}\right\rangle$ is a maximal abelian ideal and $\mathcal{E}=\left.a d\left(e_{0}\right)\right|_{\mathbb{R}^{7}}$. We will endow this algebra with the canonical metric, the standard volume form and the spin structure determined by $\eta$.

It is obvious that a representative of each element of $\overline{\mathcal{Q} \mathcal{A}}$ can be chosen to live in the image of our map. Moreover, if two structures given by $(\mathcal{E}, \bar{\eta})$ and $\left(\mathcal{E}^{\prime}, \bar{\eta}^{\prime}\right)$ are isomorphic via $\varphi^{\prime}$, we have the following:

1. $\varphi^{\prime}\left(e_{0}\right)= \pm e_{0}$ and $\varphi=\left.\varphi^{\prime}\right|_{\mathbb{R}^{7}} \in \mathrm{O}(7)$, since $\varphi^{\prime}$ preserves the metric and the orientation.

2. Denote by $\tilde{\varphi}$ any lifting of $\varphi^{\prime}$ to $\operatorname{Spin}(8)$. Since $\left(\varphi^{\prime}\right)^{*} \Omega^{\prime}=\Omega$, we have that $\operatorname{Stab}(\Omega)=\left(\varphi^{\prime}\right)^{-1} \circ$ $\operatorname{Stab}\left(\Omega^{\prime}\right) \circ\left(\varphi^{\prime}\right)$, thus $\operatorname{Stab}(\eta)=\tilde{\varphi}^{-1} \operatorname{Stab}\left(\eta^{\prime}\right) \tilde{\varphi}$. But $\operatorname{Stab}\left(\rho(\tilde{\varphi})^{-1} \eta^{\prime}\right)=\tilde{\varphi}^{-1} \operatorname{Stab}\left(\eta^{\prime}\right) \tilde{\varphi}$, so that $\eta= \pm \rho(\tilde{\varphi})^{-1} \eta^{\prime}$

3. $\varphi \circ \mathcal{E}=\operatorname{det}(\varphi) \mathcal{E}^{\prime} \circ \varphi$, since $\varphi^{\prime}$ is an isomorphism of Lie algebras.

From now on we denote by $\left(\mathbb{R}^{8}, \mathcal{E},[\eta]\right)$ to $(\mathfrak{g}, \mathfrak{h}, g, \nu, \Omega) \in \mathcal{Q} \mathcal{A}$ where $\mathfrak{g}$ is the Lie algebra $\mathbb{R}^{8}$ with maximal abelian ideal $\mathfrak{h}=\mathbb{R}^{7}, \operatorname{ad}\left(e_{0}\right)=\mathcal{E}, g$ is the canonical metric, $\nu$ is the canonical volume form and the $\operatorname{Spin}(7)$ form $\Omega$ is determined by $[\eta]$.

Remark 7.4. To obtain an analogue of Lemma 7.3, suppressing the condition $\varphi^{\prime}(\mathfrak{h})=\mathfrak{h}^{\prime}$ in the definition of isomorphism, we have to treat separatedly the case of the Lie algebra $L_{3} \oplus \mathbb{R}^{5}$. For this purpose, define $\mathcal{E}(x)=e_{1}^{*}(x) e_{2}$ and observe that lemmas 7.2 and 7.3 allow us to suppose that any isomorphism of structures with underlying Lie algebra $L_{3} \oplus \mathbb{R}^{5}$ is represented by $\varphi^{\prime}:\left(\mathbb{R}^{8}, \lambda \mathcal{E},[\eta]\right) \rightarrow\left(\mathbb{R}^{8}, \lambda^{\prime} \mathcal{E},\left[\eta^{\prime}\right]\right)$, for some $\lambda, \lambda^{\prime} \neq 0$.

The set $\varphi^{\prime}\left(\mathbb{R}^{7}\right)$ is a codimension 1 abelian ideal, hence Lemma 7.2 guarantees that $\varphi^{\prime}\left(e_{0}\right)=\cos (\theta) e_{0}+$ $\sin (\theta) e_{1}$. Denote $\mathbb{R}^{6}=\left\langle e_{2}, \ldots, e_{7}\right\rangle$ and let $v, v^{\prime} \in \mathbb{R}^{6}$ be such that $\varphi^{\prime}(v)=-\mu \sin (\theta) e_{0}+\mu \cos (\theta) e_{1}+v^{\prime}$. Then, $0=\varphi^{\prime}\left[e_{0}, v\right]=\left[\cos (\theta) e_{0}+\sin (\theta) e_{1},-\mu \sin (\theta) e_{0}+\mu \cos (\theta) e_{1}+v^{\prime}\right]=\mu \lambda^{\prime} e_{2}$. Therefore $\mu=0, \mathbb{R}^{6}$ is $\varphi^{\prime}$-invariant and $\varphi^{\prime}\left(e_{1}\right)=\mp \sin (\theta) e_{0} \pm \cos (\theta) e_{1}$.

Denote by $\varphi_{1}$ the restriction of $\varphi^{\prime}$ to $\left\langle e_{0}, e_{1}\right\rangle$ and note that: $\lambda \varphi^{\prime}\left(e_{2}\right)=\varphi^{\prime}\left[e_{0}, e_{1}\right]=\left[\varphi^{\prime}\left(e_{0}\right), \varphi^{\prime}\left(e_{1}\right)\right]=$ $\operatorname{det}\left(\varphi_{1}\right) \lambda^{\prime} e_{2}$. Hence $\varphi^{\prime}\left(e_{2}\right)=\operatorname{det}\left(\varphi_{1}\right) \frac{\lambda^{\prime}}{\lambda} e_{2}$ and $|\lambda|=\left|\lambda^{\prime}\right|$. Then, $\varphi^{\prime}$ is determined by $\varphi_{1}$ and $\varphi_{2}=\left.\varphi^{\prime}\right|_{\mathbb{R}^{5}}$, where $\mathbb{R}^{5}=\left\langle e_{3}, \ldots, e_{7}\right\rangle$, under the conditions $\frac{\lambda^{\prime}}{\lambda} \operatorname{det}\left(\varphi_{2}\right)=1$ and $\varphi^{\prime}\left(e_{2}\right)=\operatorname{det}\left(\varphi_{1}\right) \frac{\lambda^{\prime}}{\lambda} e_{2}$.

The condition over the spinor is obviously $\eta^{\prime}= \pm \rho(\tilde{\varphi}) \eta$, where $\tilde{\varphi}$ is any lifting of $\varphi^{\prime}$ to $\operatorname{Spin}(8)$.

In the following result we describe the action which appears in Lemma 7.3.

Lemma 7.5. Under the action of $\mathrm{O}(7)$ on $\operatorname{End}\left(\mathbb{R}^{7}\right)$,

$$
\varphi \cdot \mathcal{E}=\operatorname{det}(\varphi) \varphi \circ \mathcal{E} \circ \varphi^{-1}
$$

the sets $\langle\mathrm{Id}\rangle, \operatorname{Sym}_{0}^{2}\left(\mathbb{R}^{7}\right)$ and $\Lambda^{2} \mathbb{R}^{7}$ are parametrized respectively by:

1. $[0, \infty)$,

2. $\left\{\left(\lambda_{1}, \ldots, \lambda_{7}\right): \lambda_{i} \leq \lambda_{j+1}, \sum_{j=1}^{7} \lambda_{i}=0\right\} / \sim$, where $\left(\lambda_{1}, \ldots, \lambda_{7}\right) \sim\left(-\lambda_{7}, \ldots,-\lambda_{1}\right)$,

3. $\left\{\left(\lambda_{1}, \lambda_{2}, \lambda_{3}\right): 0 \leq \lambda_{1} \leq \lambda_{2} \leq \lambda_{3}\right\}$.

Proof. The first claim is obvious and the second follows from the fact that each symmetric matrix has an oriented orthonormal basis of ordered eigenvectors. Note also that $-\operatorname{Id} \cdot \operatorname{diag}\left(\lambda_{1}, \ldots, \lambda_{7}\right)=$ $\operatorname{diag}\left(-\lambda_{7}, \ldots,-\lambda_{1}\right)$, hence $\left(\lambda_{1}, \ldots, \lambda_{7}\right)$ is related to $\left(-\lambda_{7}, \ldots,-\lambda_{1}\right)$.

If $\mathcal{E}$ is a skew-symmetric endomorphism of $\mathbb{R}^{7}$ we can find a hermitian basis in $\mathbb{C}^{7}$ of eigenvectors and the eigenvalues are of the form $\left(-\lambda_{3} i,-\lambda_{2} i, \lambda_{1} i, 0, \lambda_{1} i, \lambda_{2} i, \lambda_{3} i\right)$ with $0 \leq \lambda_{j} \leq \lambda_{j+1}$. Moreover, the 
real parts of the eigenspaces associated to $-\lambda_{j} i$ and $\lambda_{j} i$ coincide. Thus, we can find a positive oriented orthonormal basis $\left(v_{1}, w_{1}, \ldots, v_{k}, w_{k}, u_{1}, \ldots u_{7-2 k}\right)$ of $\mathbb{R}^{7}$, such that $\mathcal{E}\left(v_{j}\right)=\lambda_{j} w_{j}$ and $\mathcal{E}\left(u_{j}\right)=0$. Finally note that $\left(\lambda_{1}, \lambda_{2}, \lambda_{3}\right)$ are invariantly defined in the orbit.

In Lemma 7.3, the second factor of the product of $\mathcal{Q} \mathcal{A}$ depends on $\operatorname{Stab}(\mathcal{E})$ under the action defined by (8) and it is determined by the number of equal eigenvalues. Now we compute the invariants that we defined for $\mathrm{G}_{2}$ distributions on $\mathbb{R}^{7}$ :

Proposition 7.6. Consider $\left(\mathbb{R}^{8}, \mathcal{E},[\eta]\right) \in \mathcal{Q} \mathcal{A}$ and decompose $\mathcal{E}$ according to the $\mathrm{G}_{2}$ structure induced by $\eta$, that is $\mathcal{E}=h \mathrm{Id}+E_{2}+E_{3}+E_{4}$, where $h \in \mathbb{R}, E_{2} \in \chi_{2}, E_{3} \in \chi_{3}, E_{4} \in \chi_{4}$ and $E_{4}(X)=X \times E$ for some $E \in \mathbb{R}^{7}$. Define $\Psi, \beta_{3} \in \Lambda^{3} T^{*} \mathbb{R}^{7}$ by $\Psi=\left.\Omega\right|_{\mathbb{R}^{7}}$ and $\left.\beta_{3}(X, Y, Z)=\operatorname{alt}\left(i\left(E_{3}(\cdot)\right) \Psi\right)\right]$. We have:

$$
\begin{aligned}
(* d \Omega)_{48} & =\frac{2}{7}\left(6 i(E) e^{0} \wedge \Psi-\frac{9}{4} i(E) *_{\mathbb{R}^{7}} \Psi\right)+6 \beta_{3}, \\
(* d \Omega)_{8} & =-\left(\frac{12}{7} E+4 h e_{0}\right)\left(e^{0} \wedge \Psi+*_{\mathbb{R}^{7}} \Psi\right) .
\end{aligned}
$$

Proof. The result follows immediately from Proposition 6.8 once we check that: $\mu=-\frac{1}{2} h, A_{2}=0$, $A_{3}=-\frac{1}{2} E_{3}, A=0$ and $U=-\frac{3}{2} E$.

To obtain this, first observe that $\nabla^{\mathfrak{h}} \eta=0$ and $\mathcal{L}=0$ because $\mathfrak{h}$ is an abelian ideal. From the formula of the Weingarten operator we get: $\mathcal{W}=h \mathrm{Id}+E_{3}$. To compute $U$ we use again equation (6), obtaining that:

$$
\nabla_{N} \eta=\frac{3}{2} e_{0} E \eta
$$

since $\nabla_{e_{0}} e_{0}=0$ because $\mathfrak{h}$ is an ideal and $\nabla_{e_{0}} e_{j}=\left(E_{2}+E_{4}\right)\left(e_{j}\right)$ if $j>0$.

In the next result we characterise in terms of Lemma 7.5 the type of Spin(7) structure on quasi abelian Lie algebras. For this purpose, recall that a Lie algebra is called unimodular if the volume form is not exact. In the case of the Lie algebra $\left(\mathbb{R}^{8}, \mathcal{E}\right)$, it is equivalent to say that $\mathcal{E}$ is traceless.

Theorem 7.7. Consider the Lie algebra $\left(\mathbb{R}^{8}, \mathcal{E}\right)$ endowed with the standard metric and volume form. Denote by $\mathcal{E}_{13}$ and $\mathcal{E}_{24}$ the symmetric and skew-symmetric parts of the endomorphism $\mathcal{E} \neq 0$. Then, the Lie algebra admits a $\operatorname{Spin}(7)$ structure of type:

1. parallel, if and only if $\mathcal{E}_{13}=0$ and $\mathcal{E}_{24}$ is associated to $\left(\lambda_{1}, \lambda_{2}, \lambda_{1}+\lambda_{2}\right)$ with $0 \leq \lambda_{1} \leq \lambda_{2}, \lambda_{2}>0$ as in Lemma 7.5.

2. locally conformally parallel and non-parallel if and only if $\mathcal{E}_{13}=h \mathrm{Id}$ with $h \neq 0$ and $\mathcal{E}_{24}$ is associated to $\left(\lambda_{1}, \lambda_{2}, \lambda_{1}+\lambda_{2}\right)$ with $0 \leq \lambda_{1} \leq \lambda_{2}$, as in Lemma 7.5.

3. balanced if and only if it is unimodular and $\mathcal{E}_{24}$ is associated to $\left(\lambda_{1}, \lambda_{2}, \lambda_{1}+\lambda_{2}\right)$ with $0 \leq \lambda_{1} \leq \lambda_{2}$, as in Lemma 7.5.

Moreover, if $\mathcal{E}_{24} \neq 0$ then it admits a $\operatorname{Spin}(7)$ structure of mixed type.

Proof. We identify $\mathcal{E}_{24}$ with a 2 -form $\gamma$ which can be written with respect to a positive oriented orthonormal basis $\left(X_{1}, \ldots, X_{7}\right)$ of $\mathbb{R}^{7}$ as $\gamma=\lambda_{1} X^{23}+\lambda_{2} X^{45}+\lambda_{3} X^{67}$, where $0 \leq \lambda_{j} \leq \lambda_{j+1}$ and $X^{i j}=X_{i}^{*} \wedge X_{j}^{*}$.

Due to Proposition 7.6, to prove the first part we have to check that under the assumption $\mathcal{E}_{24} \neq 0$, the existence of a spinor $\eta$ such that $\gamma \eta=0$ is equivalent to the fact that $\mathcal{E}_{24}$ is associated to $\left(\lambda_{1}, \lambda_{2}, \lambda_{1}+\lambda_{2}\right)$ with $0 \leq \lambda_{1} \leq \lambda_{2}$. This spinor exists if and only if $\rho_{7}\left(\lambda_{1} X_{2} X_{3}+\lambda_{2} X_{4} X_{5}+\lambda_{3} X_{6} X_{7}\right)$ is non-invertible for some 8-dimensional real irreducible representation $\rho_{7}: \mathrm{Cl}_{7} \rightarrow \operatorname{End}\left(\mathbb{R}^{8}\right)$ which maps the volume form $\nu_{7}$ to the identity, since they are all equivalent [15, Proposition 5.9].

It is known that the two distinct irreducible representations of $\mathrm{Cl}_{7}$ can be constructed from the octonions $\mathbb{O}\left[15\right.$, p. 51]. Specifically, those are the extension to $\mathrm{Cl}_{7}$ of the maps $\rho_{\theta}: \mathbb{R}^{7} \rightarrow \operatorname{End}\left(\mathbb{R}^{8}\right)$, $\rho_{\theta}(v)(x)=\theta v x$, where $\theta= \pm 1$ and $\mathbb{R}^{7}$ is viewed as the imaginary part of the octonions. Define the isometry $\varphi$ of $\mathbb{R}^{7}$ which maps $X_{i}$ to $e_{i}$ and note that the volume form is fixed by the extension of $\varphi$ to the Clifford algebra. The extensions of $\rho_{\theta}$ and $\varphi$ to $\mathrm{Cl}_{7}$ are denoted in the same way. We check the previous condition using the representation $\rho_{7}=\rho_{\theta} \circ \varphi: \mathrm{Cl}_{7} \rightarrow \operatorname{End}\left(\mathbb{R}^{8}\right)$, taking $\theta$ such that $\rho_{\theta}\left(\nu_{7}\right)=\operatorname{Id}$. The determinant of $\rho_{7}\left(\lambda_{1} X_{2} X_{3}+\lambda_{2} X_{4} X_{5}+\lambda_{3} X_{6} X_{7}\right)$ is given by:

$$
\left(\lambda_{1}+\lambda_{2}+\lambda_{3}\right)^{2}\left(\lambda_{1}+\lambda_{2}-\lambda_{3}\right)^{2}\left(\lambda_{1}-\lambda_{2}-\lambda_{3}\right)^{2}\left(\lambda_{1}-\lambda_{2}+\lambda_{3}\right)^{2} .
$$

Since $\lambda_{1} \leq \lambda_{2} \leq \lambda_{3}$, the endomorphism is non-invertible if and only if $\lambda_{3}=\lambda_{2}+\lambda_{1}$.

Finally, if $\mathcal{E}_{24} \neq 0$ then $\rho_{7}\left(\lambda_{1} X_{2} X_{3}+\lambda_{2} X_{4} X_{5}+\lambda_{3} X_{6} X_{7}\right) \neq 0$ so that there is a spinor inducing a $\operatorname{Spin}(7)$ structure of mixed type. 
Recall that solvmanifolds are compact quotients $G / \Gamma$, where $G$ is a simply connected solvable Lie group and $\Gamma$ is a discrete lattice. This forces the Lie algebra $\mathfrak{g}$ of $G$ to be unimodular [19, Lemma 6.2]. Thefore, using Proposition 7.6, we conclude the following:

Corollary 7.8. There exists no quasi abelian solvmanifold with an invariant locally conformally parallel and non-parallel $\operatorname{Spin}(7)$ structure.

Of course, a torus is solvmanifold which admits a parallel $\operatorname{Spin}(7)$ structure.

Corollary 7.9. If $\left(\mathbb{R}^{8}, \mathcal{E}\right)$ is a quasi abelian Lie algebra such that $\mathcal{E}$ is skew-symmetric, then it is flat. In particular, quasi abelian Lie algebras which admit an invariant parallel Spin(7) structure are flat.

Proof. Let $\left(\mathbb{R}^{8}, \mathcal{E}\right)$ be a quasi abelian Lie algebra and denote by $\mathcal{E}_{13}$ and $\mathcal{E}_{24}$ the symmetric and skewsymmetric parts of $\mathcal{E}$. It is straightforward to check that if $i, j>0$ then:

$$
\nabla_{e_{0}} e_{0}=0, \quad \nabla_{e_{0}} e_{j}=\mathcal{E}_{24}\left(e_{j}\right), \quad \nabla_{e_{i}} e_{0}=-\mathcal{E}_{13}\left(e_{i}\right), \quad \nabla_{e_{i}} e_{j}=g\left(\mathcal{E}_{13}\left(e_{i}\right), e_{j}\right) e_{0} .
$$

From this, one can deduce that if $i, j, k>0$, then the curvature tensor is given by:

$$
\begin{aligned}
& R\left(e_{0}, e_{j}\right) e_{0}=-\left(\mathcal{E}_{24} \circ \mathcal{E}_{13}+\mathcal{E}_{13} \circ \mathcal{E}_{24}\right)\left(e_{j}\right), \\
& R\left(e_{0}, e_{j}\right) e_{k}=-g\left(\mathcal{E}_{13}\left(e_{k}\right),\left(\mathcal{E}+\mathcal{E}_{24}\right)\left(e_{j}\right)\right) e_{0}, \\
& R\left(e_{i}, e_{j}\right) e_{0}=0 \\
& R\left(e_{i}, e_{j}\right) e_{k}=g\left(\mathcal{E}_{13}\left(e_{j}\right), e_{k}\right) \mathcal{E}_{13}\left(e_{i}\right)-g\left(\mathcal{E}_{13} e_{i}, e_{k}\right) \mathcal{E}_{13}\left(e_{j}\right) .
\end{aligned}
$$

Therefore, if $\mathcal{E}$ is skew-symmetric then the Lie group is flat.

Examples. Let $\mathfrak{g}$ be a quasi abelian Lie algebra determined by an endomorphism $\mathcal{E}$. Consider the unique simply connected Lie group $G$ whose Lie algebra is $\mathfrak{g}$. The split exact sequence of Lie algebras $0 \rightarrow \mathfrak{h} \rightarrow$ $\mathfrak{g} \rightarrow \mathfrak{g} / \mathfrak{h} \rightarrow 0$ lifts to a split exact sequence of Lie groups $0 \rightarrow\left(\mathbb{R}^{7},+\right) \rightarrow G \rightarrow\left(G / \mathbb{R}^{7}=\mathbb{R},+\right) \rightarrow 0$. This splitting and the conjugation $\epsilon$ on $G$ by the elements of $(\mathbb{R},+)$, provide an isomorphism $(\mathbb{R},+) \ltimes_{\epsilon}\left(\mathbb{R}^{7},+\right)$. Therefore $\left.\frac{d}{d t}\right|_{t=s} d(\epsilon(t))=s \mathcal{E}$, so that $d(\epsilon(t))=\exp (t \mathcal{E})=\epsilon(t)$, using that the exponential of $\mathbb{R}^{7}$ is the identity.

A nilmanifold with a balanced and a mixed $\operatorname{Spin}(7)$ structure. Define the endomorphism of $\mathbb{R}^{7}$

$$
\mathcal{E}=\left(\begin{array}{ccccccc}
0 & -1 & 0 & 0 & 0 & 0 & 0 \\
0 & 0 & -2 & 0 & 0 & 0 & 0 \\
0 & 0 & 0 & 0 & 0 & 0 & 0 \\
0 & 0 & 0 & 0 & -1 & 0 & 0 \\
0 & 0 & 0 & 0 & 0 & -1 & 0 \\
0 & 0 & 0 & 0 & 0 & 0 & -1 \\
0 & 0 & 0 & 0 & 0 & 0 & 0
\end{array}\right)
$$

and consider the quasi abelian Lie algebra $\left(\mathbb{R}^{8}, \mathcal{E}\right)$. Note that this is a nilpotent Lie algebra with structure equations $(0,02,03,04,05,06,07,0)$, using Salamon notation [21].

The symmetric part of $\mathcal{E}$ is traceless and the eigenvalues of its skew-symmetric part are of the form $\left(\lambda_{1}, \lambda_{2}, \lambda_{1}+\lambda_{2}\right)$. Therefore, Theorem 7.7 guarantees the existence of an invariant Spin(7) structure of type balanced and other invariant $\operatorname{Spin}(7)$ structure which is mixed. To avoid computing the eigenvalues, one can observe that it we take the standard form $\Omega_{0}$ in $\mathbb{R}^{8}$, determined by a spinor $\eta$, it holds that $e_{2} e_{3} \eta=-e_{4} e_{5} \eta=-e_{6} e_{7} \eta$ and $e_{1} e_{2} \eta=-e_{5} e_{6} \eta$. Therefore, if we identify the skew-symmetric part of $\mathcal{E}$ with a 2 -form, $\gamma$, we get that $\gamma \eta=0$.

On some nilpotent Lie algebras, the existence of a lattice is guaranteed by general theorems [16]. This case is really simple and we can compute it explicitly. The matrix of the endomorphism $\exp (t \mathcal{E})$ is:

$$
\left(\begin{array}{ccccccc}
1 & -t & t^{2} & 0 & 0 & 0 & 0 \\
0 & 1 & -2 t & 0 & 0 & 0 & 0 \\
0 & 0 & 1 & 0 & 0 & 0 & 0 \\
0 & 0 & 0 & 1 & -t & \frac{t^{2}}{2} & -\frac{t^{3}}{6} \\
0 & 0 & 0 & 0 & 1 & -t & \frac{t^{2}}{2} \\
0 & 0 & 0 & 0 & 0 & 1 & -t \\
0 & 0 & 0 & 0 & 0 & 0 & 1
\end{array}\right) .
$$


If we define $\Gamma=6 \mathbb{Z} e_{0} \times{ }_{\epsilon}\left(\mathbb{Z} e_{1} \times \mathbb{Z} e_{2} \times \cdots \times \mathbb{Z} e_{7}\right)$, then $G / \Gamma$ is a compact manifold with $\pi_{1}(G / \Gamma)=\Gamma$ which inherits both a balanced and a mixed $\operatorname{Spin}(7)$ invariant structure.

Moreover, we claim that $G / \Gamma$ is not diffeomorphic to $Q \times S^{1}$ for any 7-dimensional submanifold $Q$. Since $b_{1}(G / \Gamma)=2$, it is sufficient to prove that if a nilmanifold $G^{\prime} / \Gamma^{\prime}$ is diffeomorphic to $Q \times S^{1}$ then, $b_{1}\left(Q \times S^{1}\right) \geq 3$, or equivallently, $b_{1}(Q) \geq 2$. This assertion turns out to be true because we can check that $Q$ is homotopically equivalent to a nilmanifold. On the one hand, $Q$ is an Eilenberg-MacLance space $K\left(1, \pi_{1}(Q)\right)$, because $G^{\prime}$ is contractible. On the other hand a group is isomorphic to a lattice of a nilpotent Lie group if and only if it is nilpotent, torsion-free and finitely generated [20, Theorem 2.18]. Since $\Gamma^{\prime}=\pi_{1}\left(G^{\prime} / \Gamma^{\prime}\right)=\pi_{1}(Q) \times \mathbb{Z}$, both $\pi_{1}(Q)$ and $\Gamma^{\prime}$ verify the conditions listed above. Thus, there is a nilmanifold $Q^{\prime}$ such that $\pi_{1}\left(Q^{\prime}\right)=\pi_{1}(Q)$, which is an Elienberg-MacLane space $K\left(1, \pi_{1}(Q)\right)$. Therefore, $Q^{\prime}$ and $Q$ have the same homotopy type and $b_{1}(Q)=b_{1}\left(Q^{\prime}\right) \geq 2$, because $Q^{\prime}$ is a nilmanifold.

A compact manifold with a parallel and a mixed $\operatorname{Spin}(7)$ structure. Take the same spinor and basis of $\mathbb{R}^{7}$ as the previous example. Consider the skew-symmetric endomorphism such that $\mathcal{E}\left(e_{2}\right)=e_{3}$, $\mathcal{E}\left(e_{4}\right)=e_{5}$ and $\mathcal{E}(X)=0$ on $\left\langle e_{2}, e_{3}, e_{4}, e_{5}\right\rangle^{\perp}$. The rank of this matrix is two and it is associated to $(0,1,1)$. Therefore, Theorem 7.7 guarantees the existence of a parallel invariant Spin(7) structure and other invariant $\operatorname{Spin}(7)$ structure which is mixed. The matrix of the endomorphism $\exp \left(t \mathcal{E}_{2}\right)$ in the previous basis is:

$$
\left(\begin{array}{ccccccc}
1 & 0 & 0 & 0 & 0 & 0 & 0 \\
0 & \cos (t) & \sin (t) & 0 & 0 & 0 & 0 \\
0 & -\sin (t) & \cos (t) & 0 & 0 & 0 & 0 \\
0 & 0 & 0 & \cos (t) & -\sin (t) & 0 & 0 \\
0 & 0 & 0 & \sin (t) & \cos (t) & 0 & 0 \\
0 & 0 & 0 & 0 & 0 & 1 & 0 \\
0 & 0 & 0 & 0 & 0 & 0 & 1
\end{array}\right) .
$$

If $t \in \pi \mathbb{Z}$, the previous matrix has integers coefficients so that $\gamma=\pi \mathbb{Z} e_{0} \times_{\epsilon}\left(\mathbb{Z} e_{1} \times \mathbb{Z} e_{2} \times \cdots \times \mathbb{Z} e_{7}\right)$ is a subgroup. Moreover, $G / \Gamma$ is a compact manifold with $\pi_{1}(G / \Gamma)=\Gamma$ and inherits from $G$ both a parallel invariant $\operatorname{Spin}(7)$ structure and a mixed invariant one.

According to Remark 7.9, this manifold is flat. It is the mapping torus of $\exp (\pi \mathcal{E}): X \rightarrow X$, where $X$ is a 7 torus. Indeed, since $\exp (\pi \mathcal{E})^{2}=\mathrm{Id}$, the 8 -torus is a 2 -fold connected covering of $G / \Gamma$.

\section{ACKNOWLEDGEMEnTs}

I would like to thank my thesis directors, Giovanni Bazzoni and Vicente Muñoz, for useful conversations, advices and encouragement.

The author is supported by a grant from Ministerio de Educación, Cultura y Deporte, Spain (FPU16/03475).

\section{REFERENCES}

[1] I. Agricola, S. Chiossi, T. Friedrich, J. Holl, Spinorial description of SU(3) and $\mathrm{G}_{2}$-manifolds, J. Geom. Phys., 98 (2015): 535-555.

[2] M. Berger. Sur les groupes d' holonomie homogènes de variétés à conexion affine et des variétés riemanniennes, Bull. Soc. Math. France, 283 (1955): 279-330.

[3] R. L. Bryant, Metrics with exceptional holonomy, Ann. Math, 126 (1987): 525-576.

[4] R. L. Bryant, S. M. Salamon, On the construction of some complete metrics with exceptional holonomy, Duke Math. J. 58 (1989): 829-850.

[5] S. Chiossi, S. Salamon, The intrinsic torsion of $\mathrm{SU}(3)$ and $\mathrm{G}_{2}$ structures, Differential Geometry, Valencia 2001, World Sci. Publ., (2002) 115-133.

[6] M. Fernández, A classification of Riemannian manifolds with structure group Spin(7), Ann. Mat. Pura Appl. 143 (1986): 101-122.

[7] M. Fernández and A. Gray, Riemannian manifolds with structure group $\mathrm{G}_{2}$, Ann. Mat. Pura Appl., 132 (1982), $19-45$.

[8] T. Friedrich, Dirac Operators in Riemannian geometry, Graduate studies in mathematics. American Mathematical Society, 2000.

[9] T. Friedrich, On types of non-integrable geometries, Rend. Circ. Mat. Palermo, 71 (2003):99-113.

[10] T. Friedrich, S. Ivanov, Parallel spinors and connections with skew-symmetric torsion in string theory, Asian J. Math. 6(2002): 303-336.

[11] S. Ivanov, Connections with torsion, parallel spinors and geometry of Spin(7)-manifolds, Math. Res. Lett, 2-3 (2004): $171-186$.

[12] D. Joyce, Compact Riemannian 7-manifolds with holonomy $\mathrm{G}_{2}$ I, J. Diff. Geom., 43 (1996): 291-328.

[13] D. Joyce, Compact Riemannian 7-manifolds with holonomy $\mathrm{G}_{2}$ II, J. Diff. Geom., 43 (1996): 329-375.

[14] D. Joyce, Compact 8-manifolds with holonomy Spin(7), Invent. Math., 123 (1996): 507-552.

[15] H.B. Lawson, M.L.Michelsohn, Spin geometry, Princeton mathematical series 38, Princeton University Press, 1989. 
[16] A.I. Malcev, On a class of homogeneous spaces.Reprinted in Amer. Math. Soc. Translations Series 1, 9 (1962): 276307.

[17] Martín-Cabrera, Orientable Hypersurfaces of Riemannian Manifolds with Spin(7)-Structure, F. Acta Mathematica Hungarica, 76(1997): 235.

[18] Martín-Cabrera, Spin (7)-structures in principal fibre bundles over Riemannian manifolds with $\mathrm{G}_{2}$-structure, Rend. Circ. Mat. Palermo, 44 (1995): 249.

[19] J. Milnor, Curvatures of left invariant metrics on Lie groups, Advances Math., 21(1976): 293-329.

[20] M. S. Raghunathan, Discrete subgroups of Lie groups, Springer-Verlag Berling Heidelberg New-York, 1972.

[21] D. Salamon, Complex structures on nilpotent Lie algebras, J. Pure appl. Algebra, 157(2001): 311-333.

[22] D. Salamon, T. Walpuski. Notes on the octonions, https://arxiv.org/abs/1005.2820.

Departamento de Álgebra, Geometría y Topología, Universidad Complutense de Madrid, Plaza de Ciencias 3, 28040 MADRID, Spain

E-mail address: Immerchan@ucm.es 